\title{
Dissociation equilibrium height and friction coefficient in pipeline transportation of gas hydrate-bearing sediment particles
}

\author{
Peng $\mathrm{Li}^{\mathrm{a}}$, Xuhui Zhang ${ }^{\mathrm{a}, \mathrm{b}, *}$, Xiaobing $\mathrm{Lu}^{\mathrm{a}}{ }^{\mathrm{a}} \mathrm{b}$ \\ ${ }^{a}$ Institute of Mechanics, Chinese Academy of Sciences, Beijing, 100190, China \\ ${ }^{\mathrm{b}}$ School of Engineering Science, University of Chinese Academy of Sciences, Beijing, 100049, China
}

\section{A R T I C L E I N F O}

\section{Keywords:}

Gas hydrate

Dissociation equilibrium height

Friction coefficient

Power-law correlation

Gas-liquid-solid flow

Dimensional analysis

\begin{abstract}
A B S T R A C T
Compared with traditional fossil fuels, gas hydrates have vast reserves, cause little pollution, and are an essential strategic energy resource. Pipeline transportation is critical to realize the development and utilization of natural gas hydrate resources. The Eulerian multiphase flow model within the computational fluid dynamics software FLUENT was employed to calculate gas-liquid-solid flow, taking into account phase interactions, heat transfer, and collision among particles. Pipeline transportation of gas hydrate-bearing sediment (GHBS) particles was studied based on dimensional analysis and numerical simulation. First, the essential dimensionless numbers controlling multiphase flow and hydrate dissociation were deduced. Further, the obtained simulation results clearly indicate that when the system was in a stable state, there was a dissociation equilibrium height above which hydrate dissociated completely. The influences of the dimensionless numbers on the dissociation equilibrium height and friction coefficient in the pipe were determined, and power-law correlations for the dissociation equilibrium height and friction coefficient were obtained from the numerical data. Finally, an analytical expression of dissociation equilibrium height was derived by decoupling the solid-liquid flow and gas hydrate dissociation, and the validity of the power-law correlation for the dissociation equilibrium height was verified by the analytical expression.
\end{abstract}

\section{Introduction}

In recent years, natural gas hydrate (GH) has attracted increasing attention because of its large reserves and low pollution compared with fossil fuels. To data, known methods for hydrate exploitation consist of the thermal stimulation method, depressurization method, $\mathrm{CO}_{2^{-}}$ replacement method, and chemical injection method (Moridis et al., 2009; Makogon and Omelchenko, 2013; Sivla and Dawe, 2011; White et al., 2011; Zhang and Lu, 2016). Trial production of hydrates has demonstrated that these methods can dissociate GH through the interference of temperature and pressure. However, it is difficult to meet the development efficiency requirements of commercial development (Collett et al., 2011; Fujii et al., 2013; Makagon et al., 2005). Considering the low heat transfer and mining efficiency of the traditional exploitation methods, Zhang and Lu (2014) proposed a new method called mechanical-thermal exploitation.

The admirable method for improving the heat transfer efficiency during GH exploitation is to reduce the path of heat transfer and increase the surface area of heat transfer. The main feature of mechanical-thermal exploitation is to utilize the convective heat transfer between the warm seawater and the gas hydrate-bearing sediment (GHBS) particles. The first step of mechanical-thermal exploitation is to excavate the underground GH formation like coal mining, as shown in Fig. 1. The produced sediments are cut into small particles, which are then mixed with the warmer seawater and transported through a vertical pipe to the storage box on the sea surface. During uplifting, GH dissociates with the decrease of pressure and the increase of temperature, the soils are separated and backfilled.

To excavate and crush GHBS into particles, a submarine excavator or high-pressure water gun can be used. Based on the working principle of cutter suction dredger and deep-sea manganese nodule mining, Wu et al. (2017) designed a marine GH mining tool, and the rapid poly ballast and efficient chip were achieved. Besides, Wang et al. (2017) proposed a new technical idea of GH jet breaking exploitation without changing the temperature and pressure conditions of GH formations and designed the matching nozzle tools for $\mathrm{GH}$ jet breaking. All these studies provide a potential practice for the deep-sea hydrate excavation.

The seawater lifting technology was first applied in coal mining, and

\footnotetext{
* Corresponding author. Institute of Mechanics, Chinese Academy of Sciences, Beijing, 100190, China.

E-mail addresses: lipeng@imech.ac.cn (P. Li), zhangxuhui@imecha.ac.cn (X. Zhang), xblu@imech.ac.cn (X. Lu).
} 


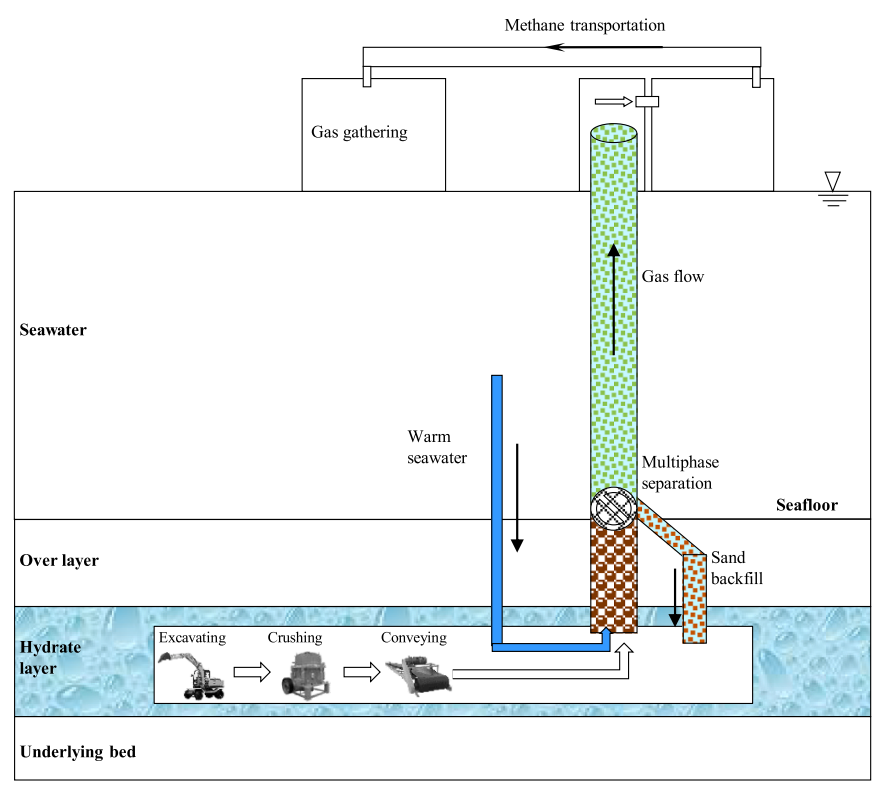

Fig. 1. Schematic diagram of mechanical-thermal hydrate exploitation.

now it has been successfully used in the trial mining of deep-sea manganese nodules, providing its feasibility in the development of marine resources. During lifting of the GHBS particles in the pipe, the warm seawater from the upper depth is transported downwards through a pump to mix with GHBS particles and then uplift upwards. Convective heat transfer between GHBS particles and water leads to GH dissociation, releasing gas into the water. The hydrate is anticipated to reach complete dissociation when the particles come to a certain height, where a multiphase separation system is set up to capture and backfill the remaining sand particles and control the gas upwards flow to a gathering installation (Li et al., 2019). The transportation of GHBS particles in the vertical pipe is the crucial process.

This study mainly focused on the transport process of multiphase flow coupled with GH dissociation in the vertical pipe and ignored the separation system. Here, we define the dissociation equilibrium height as the rising distance of solid particles where the hydrate dissociates completely. The magnitude of the dissociation equilibrium height determines the installation position of the separation system. The friction coefficient is defined as the ratio of friction drag per unit area of the pipe wall to the dynamic pressure at the pipe entrance. The flow in the pipe is characterized by the dissociation equilibrium height and friction coefficient. However, the multiphase flow in the pipe is characterized by a dense particle reaction system (Zhong et al., 2016) that contains multiphase flow and GH dissociation simultaneously. It is challenging to obtain optimized controlling parameters by theoretical analysis because of the complexity of the problem. Therefore, to understand the fundamental mechanisms of the multiphase flow, essential dimensionless parameters are first derived by dimensional analysis. Li et al. (2019) developed a three-dimensional numerical model to describe the coupling of gas-liquid-solid three-phase flow and GH dissociation in a vertical pipe. The critical problem of computational fluid dynamics simulation of a particle reaction system is the coupling of multiphase flow and heat and mass transfer. In this study, the same numerical method was used to study the effects of the obtained dimensionless numbers on the dissociation equilibrium height and friction coefficient. The power-law correlations for the dissociation equilibrium height and friction coefficient of the pipe were obtained using the numerical data.

Moreover, it has been found that solid-liquid flow and hydrate dissociation possess different characteristic time scales. Hydrate dissociation occurs substantially slower than the upward expansion of solid particles; thus, the two physical processes can be viewed as being decoupled. For this paper, an analytical expression of dissociation equilibrium height was derived by means of a decoupling method, and the applicability of this expression was also evaluated.

This paper is arranged as follows. The problem formulation and dimensional analysis are introduced in Section 2. The numerical simulation method and a benchmark simulation are described in Section 3. Section 4 discusses the effects of dimensionless numbers on the transport system in the pipe and the power-law correlations for the dissociation equilibrium height and friction coefficient are obtained based on the numerical data. Section 5 gives an analytical expression of the dissociation equilibrium height and discusses its applicability. The conclusions are presented in Section 6.

\section{Problem formulation and dimensional analysis}

The physical process of the multiphase flow in a vertical pipe can be described as follows. A mixture of warmer seawater and GHBS particles are injected into the vertical pipe continuously at a certain velocity to form a fluidized bed. When flowing upward, the particles are heated by the seawater and the pressure drops. Once the hydrate phase equilibrium condition is broken, $\mathrm{GH}$ in the particles dissociates into water and methane gas. The gas produced by this dissociation occurs in a dissolved state and small bubbles. After a particular time, the GH dissociates completely. When the height corresponding to the complete dissociation of hydrate is independent of time, the system is considered to have reached a stable state, and this height is called the dissociation equilibrium height. A multiphase separation system installed at this height is then used to separate the remaining sand particles from the multiphase system. Thus, the whole pipe can be distinguished into four zones: the solid-liquid two-phase flow zone, the gas-liquid-solid flow with hydrate dissociation zone, the gas-liquid-solid flow without hydrate dissociation zone, and the upper gas-liquid flow zone, as shown in Fig. 2. Sand particle separation and upward gas flow are beyond the scope of this paper and will not be discussed here.

For the sake of simplicity, the particles are assumed to be spherical, and fragmentation of particles is not considered. The GHBS particles are composed of three components (sand, water, and GH), and the thermal properties of each component are assumed to be independent of temperature and pressure. The gas phase is presumed to be incompressible. The dissolution, coalescence, and bursting of the bubbles are ignored, and it is presumed that the diameter of the gas bubbles remains unchanged. The heat exchange between the water and the wall is also not considered.

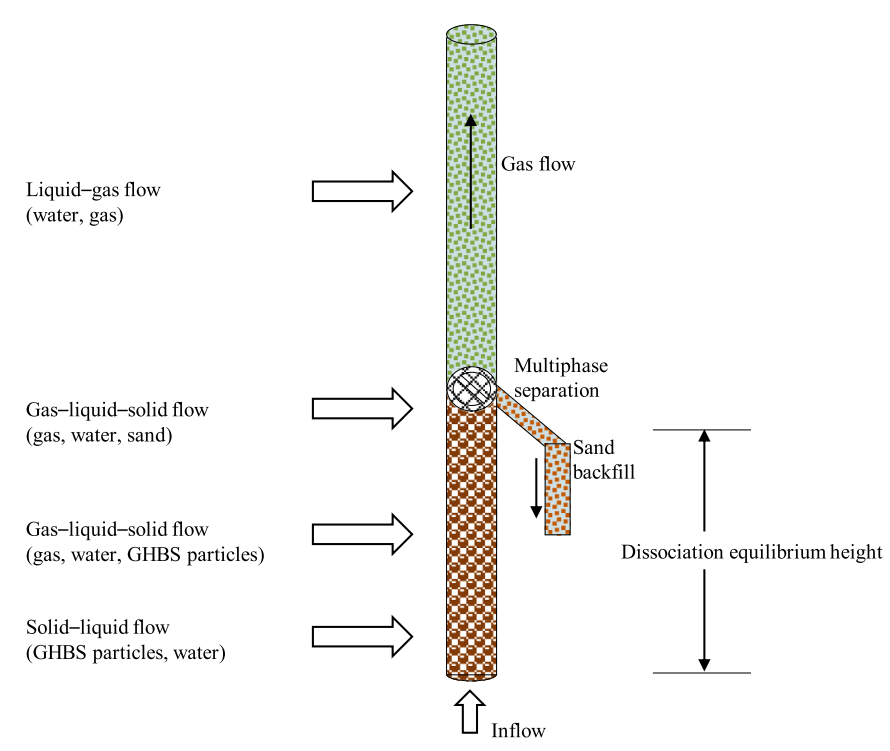

Fig. 2. Multiphase flow transport system coupled with GH dissociation in the vertical pipeline. 
Dickens and Quinby's expression (1994) for the phase equilibrium condition of GH is given by:

$\frac{1}{T_{e}}=3.79 \times 10^{-3}-2.83 \times 10^{-4} \log P_{e}$,

where $T_{e}$ is the equilibrium temperature $(\mathrm{K})$ and $P_{e}$ is the equilibrium pressure (MPa).

The rate of hydrate dissociation is expressed as (Li et al., 2018):

$r=-\frac{d C_{\mathrm{GH}}}{d t}=k C_{\mathrm{GH}}^{\eta}$,

where $r$ is the reaction rate $\left(\mathrm{kmol} / \mathrm{m}^{3} \cdot \mathrm{s}\right), k$ is the rate constant, $C_{\mathrm{GH}}$ is the concentration of non-dissociated $\mathrm{GH}\left(\mathrm{kmol} / \mathrm{m}^{3}\right)$, and $\eta$ is the rate exponent, which is dimensionless. The mass source term of GH, $S_{\mathrm{GH}}=$ - $M_{\mathrm{GH}} k C_{\mathrm{GH}}^{\eta}$, represents the mass change rate of dissociated $\mathrm{GH}$ $\left(\mathrm{kg} / \mathrm{m}^{3} \cdot \mathrm{s}\right)$, where $M_{\mathrm{GH}}$ is the molar mass of $\mathrm{GH}(\mathrm{kg} / \mathrm{kmol})$.

The rate constant is described by the Arrhenius equation:

$k=\frac{A}{\left(\pi d_{s}^{3} / 6\right)^{1-\eta}}\left(\frac{T_{s}}{T_{e}}\right)^{\beta} \exp \left(-\frac{E_{a}}{R T_{s}}\right)$,

where $A$ is the pre-exponential factor $\left(\mathrm{kmol}^{1-\eta} \cdot \mathrm{s}^{-1}\right), \beta$ is the temperature exponent, $E_{a}$ is the activation energy $(\mathrm{J} / \mathrm{mol}), R$ is the universal gas constant $(\mathrm{J} / \mathrm{mol} \cdot \mathrm{K})$, and $T_{s}$ is the temperature of the particles (K) ( $\mathrm{Li}$ et al., 2019).

The parameters controlling the multiphase flow and hydrate dissociation are listed as follows:

Water: density $\rho_{l}$, viscosity $\mu_{l}$, thermal conductivity $\lambda_{l}$, specific heat $c_{l}$, inlet water temperature $T_{l 0}$, and inlet mixture velocity $v_{0}$.

$\mathrm{GH}$ : latent heat of dissociation $\Delta H$ and dissociation rate $S_{\mathrm{GH}}$.

Methane gas: density $\rho_{g}$, viscosity $\mu_{g}$, thermal conductivity $\lambda_{g}$, specific heat $c_{g}$, initial temperature $T_{s 0}$, bubble diameter $d_{g}$, and surface tension coefficient $\sigma$.

GHBS particles: density $\rho_{s}$, thermal conductivity $\lambda_{s}$, specific heat $c_{s}$, diameter $d_{s}$, initial solid volume fraction $\alpha_{s 0}$, porosity $\varphi$, hydrate saturation $S_{H}$, and inlet solid temperature $T_{s 0}$. The thermal parameters of GHBS particles can be expressed as a linear superposition of the corresponding parameters of $\mathrm{GH}$, water, and sand:

$N_{s}=\sum_{i=1}^{3} \alpha_{i} N_{i}$

where $N$ represents $\rho_{s}, \lambda_{s}$, and $c_{s}$, and $\alpha$ is the volume fraction of each component. The subscript $i$ represents $\mathrm{GH}$, water, and sand, respectively.

Geometric parameters: pipe diameter $D$ and pipe height $H$.

Gravitational acceleration: $g$.

Because GHBS particles and gas bubbles are suspended in water, the body force should appear in the form of density difference: $\left(\rho_{s}-\rho_{l}\right) g$ and $\left(\rho_{l}-\rho_{g}\right) g$. The temperature is also expressed in the form of the temperature difference $T_{l 0}-T_{s 0}$ and $T_{l 0}-T_{g 0}$. The final steady-state of the multiphase flow system, characterized by the dissociation equilibrium height $h_{e}$ and the pressure drop between inlet and outlet $\Delta p$ is a function of the above controlling parameters:

Table 1

Dimensionless numbers corresponding to different physical processes.

\begin{tabular}{ll}
\hline Physical process & Dimensionless numbers \\
\hline $\begin{array}{l}\text { Gas-liquid-solid } \\
\text { flow }\end{array}$ & $A r_{s}=\frac{\left(\rho_{s}-\rho_{l}\right) g \rho_{l} d_{s}^{3}}{\mu_{l}^{2}}, A r_{g}=\frac{\left(\rho_{l}-\rho_{g}\right) g \rho_{l} d_{g}^{3}}{\mu_{l}^{2}}, R e_{s}=\frac{\rho_{l} v_{0} d_{s}}{\mu_{l}}, B o=$ \\
& $\frac{g\left(\rho_{l}-\rho_{g}\right) d_{g}^{2}}{\sigma}, \alpha_{s 0}, R e_{g}=\frac{\rho_{l} v_{0} d_{g}}{\mu_{l}}, \frac{\rho_{l}}{\rho_{s}}, \frac{\mu_{g}}{\mu_{l}}, \frac{d_{s}}{D}, \frac{d_{s}}{H}$ \\
Heat transfer & $\frac{T_{l 0}-T_{s 0}}{T_{s 0}}, \operatorname{Pr}=\frac{\mu_{l} c_{l}}{\lambda_{l}}, \frac{\lambda_{s}}{\rho_{s} d_{s} c_{s} v_{0}}, \frac{\lambda_{g}}{\rho_{g} d_{g} c_{g} v_{0}}$ \\
GH dissociation & $\varphi S_{H}, \frac{\lambda_{s}}{S_{\mathrm{GH}} c_{s} d_{s}^{2}}, J a=\frac{\left(T_{l 0}-T_{s 0}\right) c_{s}}{\Delta H}, \frac{\left(T_{l 0}-T_{g 0}\right) c_{g}}{\Delta H}, \frac{T_{s 0} c_{s}}{\Delta H}$ \\
\hline
\end{tabular}

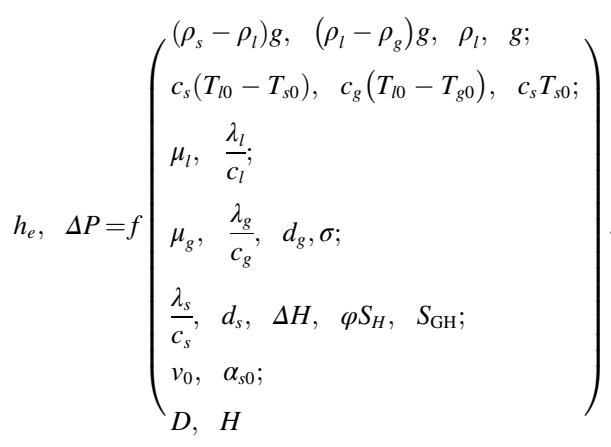

Taking $d_{s}, \rho_{l}$, and $\mu_{l}$ as a unit system produces the following dimensionless relationship:

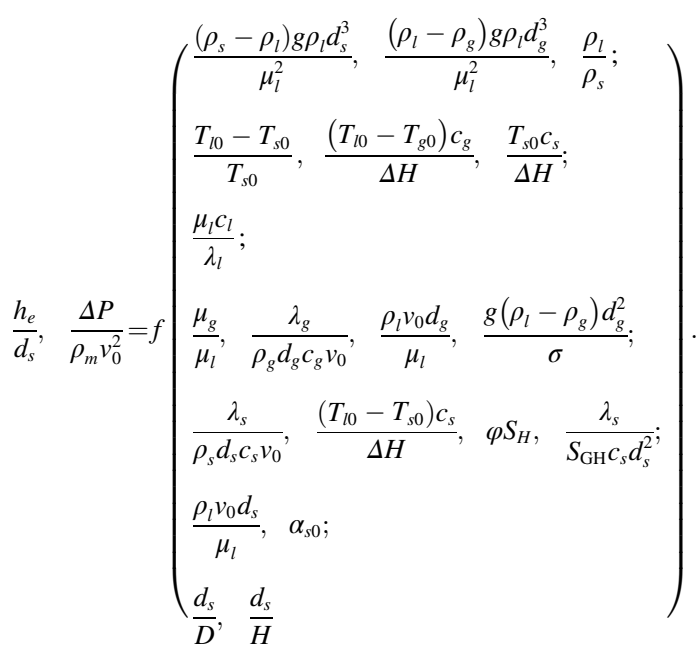

Note that $\Delta P / \rho_{m} v_{0}^{2}$ represents the relative magnitude of the pressure drop to the dynamic pressure at the inlet and can measure the magnitude of the momentum loss rate in the pipe where $\rho_{m}$ is the density of the mixture at the inlet $\rho_{m}=\alpha_{s 0} \rho_{s}+\left(1-\alpha_{s 0}\right) \rho_{l}$. Assuming that the friction drag is evenly distributed along the pipe wall, the friction drag per pipe wall unit area $\tau$ can be defined as the total drag divided by the total pipe wall area (Tan, 2011):

$\tau=\frac{\Delta P \cdot(\pi / 4) D^{2}}{\pi D H}=\frac{\Delta P}{4} \cdot \frac{D}{H}$

The friction coefficient $c_{d}$ is defined as the ratio of $\tau$ to the dynamic pressure $\rho_{m} v_{0}^{2} / 2$ :

$c_{d}=\frac{\tau}{\rho_{m} v_{0}^{2} / 2}=\frac{\Delta P D}{2 \rho_{m} v_{0}^{2} H}$.

The above 19 derived dimensionless numbers are classified according to different physical processes, as shown in Table 1.

\section{(i) Multiphase flow process}

In the process of the mixture of GHBS particles and warm water entering the vertical pipe, the water lifting capacity can be characterized by the Reynolds number $R e_{s}=\rho_{l} v_{0} d_{s} / \mu_{l}$ and the Archimedes number $A r_{s}=\left(\rho_{s}-\rho_{l}\right) g \rho_{l} d_{s}^{3} / \mu_{l}^{2}$ characteristics the particle sedimentation effect. The ratio $R e_{s} / A r_{s}=v_{0} \mu_{l} / d_{s}^{2}\left(\rho_{s}-\rho_{l}\right) g$ measures the relative magnitude of the water lifting capacity to the particle sedimentation effect. When the flow system reaches a stable state, the effects of $R e_{s}$ and $A r_{s}$ can be measured by the ratio $R e_{s} / A r_{s}$ (Li et al., 2018).

The forces induced by the water on the gas bubbles include inertial force, viscous drag, and buoyancy force. The Reynolds number $R e_{g}=$ $\rho_{l} v_{0} d_{g} / \mu_{l}$ represents the ratio of the inertia effect to the viscosity effect. The Archimedes number $A r_{g}=\left(\rho_{l}-\rho_{g}\right) g \rho_{l} d_{g}^{3} / \mu_{l}^{2}$ represents the 
buoyancy weight caused by the density difference. The Bond number $B o=g\left(\rho_{l}-\rho_{g}\right) d_{g}^{2} / \sigma$ represents the ratio of gravity and the surface tension of the gas bubbles. The bubbles move upward in water because of buoyancy and inertial forces, which further results in the change of the solid concentration distribution. If the surface tension of the gas bubbles is not considered, the change in the concentration of solid particles caused by motion of the bubbles can be characterized by $d_{g} / d_{s}$; the reasonableness of this hypothesis is confirmed by a-posteriori calculations in Section 4.6.

\section{(ii) Heat transfer process}

When solid particles move in water at a relative velocity $\Delta v$, the total heat transfer process comprises heat conduction inside the sediment particles and heat convection in water (Fig. 3). The dimensionless number

$\frac{\lambda_{s}}{\rho_{s} d_{s} c_{s} v_{0}}=\frac{d_{s}}{v_{0}} / \frac{\rho_{s} c_{s} d_{s}^{2}}{\lambda_{s}}=\frac{t_{\mathrm{c}, \text { flow }}}{t_{\mathrm{c}, \text { cond }}}$

represents the ratio of the characteristic time of water flowing through a particle surface $t_{\mathrm{c} \text {,flow }}$ to the characteristic time of heat conduction inside the particle $t_{\mathrm{c}, \text { cond }}$. Similarly, the dimensionless number

$\frac{\lambda_{g}}{\rho_{g} d_{g} c_{g} v_{0}}=\frac{d_{g}}{v_{0}} / \frac{\rho_{g} c_{g} d_{g}^{2}}{\lambda_{g}}$

represents the ratio of the two characteristic times of the heat transfer between the water and gas bubbles. Here, we focus on the heat transfer between water and particles.

The Nusselt number $N u_{s}$ provides a measure of the convective heat transfer occurring at the surface of a particle. Generally, the Nusselt number $N u_{s}$ can be expressed as a function of Reynolds number $R e_{s}$ and the Prandtl number $\mathrm{Pr}=\mu_{l} c_{l} / \lambda_{l}$,

$N u_{s}=f\left(R e_{s}, \operatorname{Pr}\right)$.

If the thermal physical parameters of the water are assumed to be constant, then $P r$ is a constant, and $N u_{s}$ is determined only by $R e_{s}$.

(iii) Hydrate dissociation process

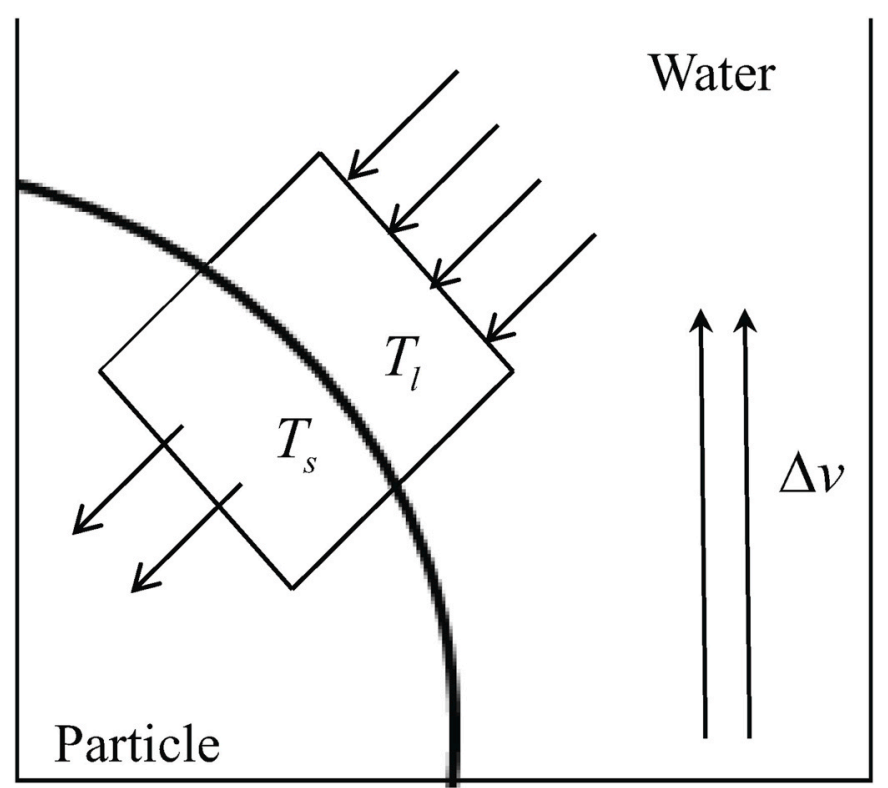

Fig. 3. The heat transfer process between a sediment particle and ambient water.
From the definition of the hydrate dissociation rate, the characteristic time for hydrate dissociation can be expressed as:

$t_{\mathrm{c}, \mathrm{dis}}=\frac{\rho_{\mathrm{GH}}}{S_{\mathrm{GH}}}$.

The dimensionless number $\lambda_{s} / S_{\mathrm{GH}} c_{s} d_{s}^{2}$ can be written in the following form:

$\frac{\lambda_{s}}{S_{\mathrm{GH}} c_{s} d_{s}^{2}}=\frac{\rho_{\mathrm{GH}}}{S_{\mathrm{GH}}} / \frac{\rho_{\mathrm{GH}} c_{s} d_{s}^{2}}{\lambda_{s}}=\frac{t_{\mathrm{c}, \mathrm{dis}}}{t_{\mathrm{c}, \mathrm{cond}}}$.

Therefore, it represents the ratio of the characteristic time of hydrate dissociation $t_{\mathrm{c}, \mathrm{dis}}$ to the characteristic time of hydrate heat conduction $t_{\mathrm{c}, \text { cond }}$.

According to Eqs. (2) and (3), the hydrate concentration and temperature difference between water and hydrate are the main factors affecting the dissociation rate. The hydrate concentration is characterized by $\varphi S_{H}$, and the temperature difference is characterized by $\left(T_{l 0}-\right.$ $\left.T_{s o}\right) / T_{s o}$. The amount of hydrate dissociation per unit time also depends on the heat transfer efficiency, which is characterized by $\mathrm{Nu}_{s}$.

The dimensionless Jakob number $J a=\left(T_{l 0}-T_{s 0}\right) c_{s} / \Delta H$ represents the ratio of sensible to latent energy absorbed during hydrate dissociation.

The material properties and particle size remain constant while $d_{g} / d_{s}$ and $d_{s} /$ Hare fixed. Therefore, the dissociation equilibrium height $h_{e}$ and the friction coefficient $c_{d}$ can be expressed as:

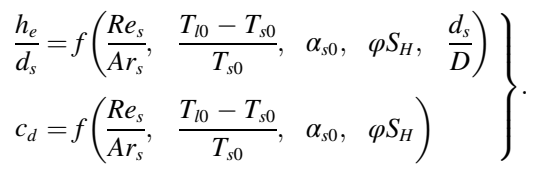

For the following sections, new data obtained from numerical simulations for the pipe transport system were collected to derive powerlaw correlations between $h_{e} / d_{s}, c_{d}$, and the prescribed parameters:

$\left.\begin{array}{l}\frac{h_{e}}{d_{s}}=a_{1}\left(\frac{R e_{s}}{A r_{s}}\right)^{b_{1}}\left(\frac{T_{l 0}-T_{s 0}}{T_{s 0}}\right)^{c_{1}}\left(\alpha_{s 0}\right)^{d_{1}}\left(\varphi S_{H}\right)^{e_{1}}\left(\frac{d_{s}}{D}\right)^{f_{1}} \\ c_{d}=a_{2}\left(\frac{R e_{s}}{A r_{s}}\right)^{b_{2}}\left(\frac{T_{l 0}-T_{s 0}}{T_{s 0}}\right)^{c_{2}}\left(\alpha_{s 0}\right)^{d_{2}}\left(\varphi S_{H}\right)^{e_{2}}\end{array}\right\}$.

Different dimensionless numbers were used in the pipe transport system; one of the dimensionless numbers varied while the others remained unchanged. For each single dimensionless number, power-law correlations of $h_{e} / d_{s}$ and $c_{d}$ were developed. The exponent of each dimensionless number was obtained by curve-fitting. Then, the predicted exponents were inserted into Eq. (15), giving rise to the average values of the pre-factors $a_{1}$ and $a_{2}$ by the least squares method. The prefactors and exponents could be adjusted to achieve the best fit for $h_{e} / d_{s}$ and $c_{d}$.

\section{Computational framework}

The details of the numerical model, including the governing equations and methods of solution, can be found in the literature ( $\mathrm{Li}$ et al., 2019). Throughout this paper, unless specified, a cylindrical pipe of diameter $\times$ height $=0.1 \mathrm{~m} \times 10 \mathrm{~m}$ was considered. Fig. 4 shows a sketch of the pipe and the mesh. The bottom of the pipe was set as the velocity inlet, and the top of the pipe was set as the pressure outlet. The outlet pressure was set to be $10 \mathrm{MPa}$ (assuming that water with 1,000 m depth pressed the outlet). The pressure difference between the inlet and the outlet was only 0.1 MPa. According to Eq. (1), the amplitude of variation of the equilibrium temperature $\Delta T_{e}$ was $0.09 \mathrm{~K}$. If the inlet liquid temperature $T_{l 0}$ is taken as $295.15 \mathrm{~K}$, then $\Delta T_{e} / T_{l 0}=3.0 \times 10^{-4} \ll 1$. Therefore, the influence of the pressure change along the vertical pipe on the GH dissociation rate was not considered in this study. The equilibrium temperature was taken as the temperature corresponding to the 


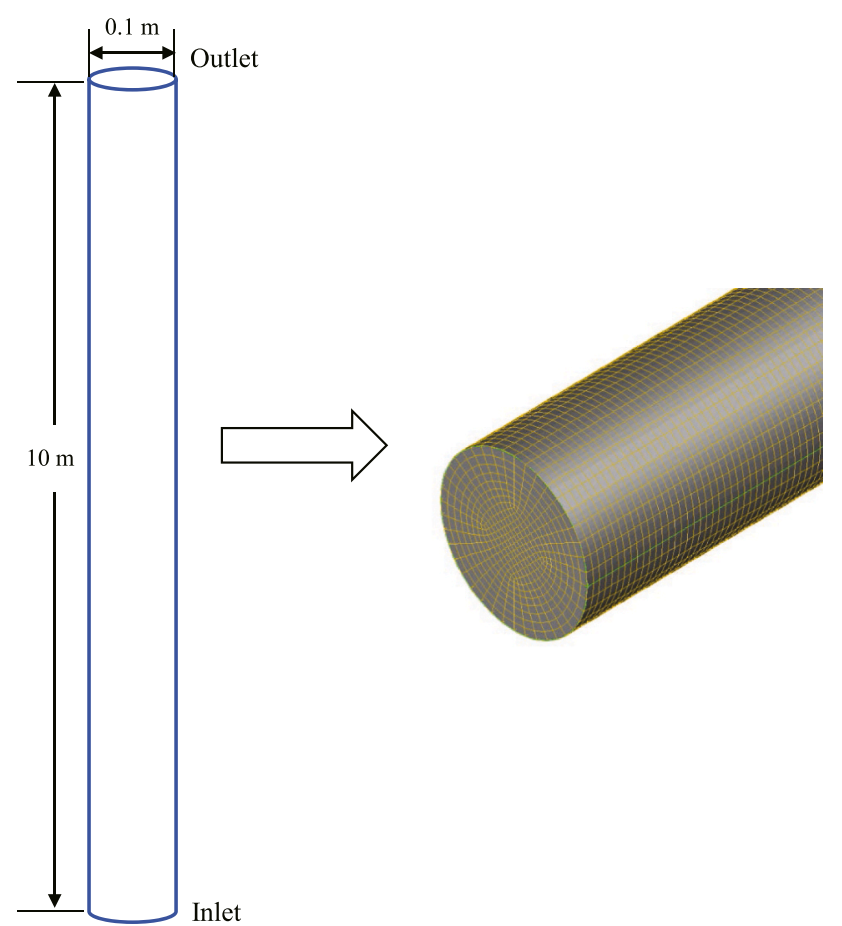

Fig. 4. Sketch of the pipe and structured grid.

outlet pressure (10 MPa), $T_{e}=285.15 \mathrm{~K}$. The water initially occupied the whole pipe. Gravity acted in the negative z-direction. First, a base case was analyzed to deepen our understanding of the dissociation and flow process of GHBS particles in the pipe. Then, as described in Section 4 , a parametric study of the process was performed and the correlations were obtained.

For this section, a base case simulation was performed with the properties of the three gas-liquid-solid three phases shown in Table 2. The viscosity, specific heat, and thermal conductivity of the three phases were kept constant in all cases. Taking $v_{0}=0.20 \mathrm{~m} / \mathrm{s}, T_{g 0}=285.15 \mathrm{~K}$, $T_{l 0}=295.15$ Kand $T_{s 0}=280.15 \mathrm{~K}$, consequently, $R e_{s} / A r_{s}=2.23 \times$ $10^{-3},\left(T_{l 0}-T_{s 0}\right) / T_{s 0}=0.054, d_{s} / D=0.030, d_{g} / d_{s}=0.10$, and $d_{s} / H=$ $3 \times 10^{-4}$. The porosity of GHBS particles was assumed to be $\varphi \equiv 0.40$, and the saturation was set as $S_{H}=0.40$, such that $\varphi S_{H}=0.16$. The initial solid volume fraction was taken as $\alpha_{s 0}=0.10$. The kinetic parameters of hydrate dissociation were set as: $\eta=0.6, A=3.89 \times$ $10^{12} \mathrm{kmol}^{1-\eta} \cdot \mathrm{s}^{-1}, \beta=2, E_{a}=9.83 \times 10^{4} \mathrm{~J} / \mathrm{mol}, R=8.314 \mathrm{~J} / \mathrm{mol}$. $\mathrm{K}$, and $\Delta H=54.5 \mathrm{~kJ} / \mathrm{mol}$.

As shown in Fig. 5a, the height corresponding to the complete dissociation of hydrate experienced an increase-decrease process before it reached a constant steady value. It increased monotonously before $t^{*}=3.54$ because the mixture of particles and water continued to enter the pipe and the height of particles increased. At $t^{*}=3.54$, a portion of the GHBS particles at the top of the pipe completely dissociated; the corresponding height can be called the dissociation completion height, denoted by $h$. Portions of the particles and gas were carried out of the pipe because of the limited height of the pipe after $t^{*}=3.54$; thus, $h$ drastically decreased from the peak. After a period of time, $h$ eventually leveled off to the equilibrium height $h_{e}$. It was found that $h_{e} / d_{s}=$ $2,506.67$ for the base case.

Fig. $5 \mathrm{~b}$ shows the time evolution of $c_{d}$. It increased to the maximum value before the particles flowed out of the pipe $\left(t^{*}=3.54\right)$, and fluctuated severely as the volume of the gas increased. After $t^{*}=3.54$, it drastically decreased to a constant value. Fig. 6 also shows that $h_{e}$ and $c_{d}$ presented similar trends over time. For the base case, $c_{d}=13.41$ at the steady state.

\section{Effect of dimensionless numbers and correlations for the pipe transport system}

The effects of the various dimensionless parameters on $h_{e} / d_{s}$ and $c_{d}$ were characterized by perturbing them about the base case, and correlations between $h_{e} / d_{s}, c_{d}$, and the prescribed dimensionless numbers were obtained. All the computed values of $v_{0}, T_{l 0}, \alpha_{s 0}, S_{H}, D$, and the corresponding dimensionless numbers are given in Table 3 . Throughout this section, $T_{e} \equiv 285.15 \mathrm{~K}, \quad T_{s o} \equiv 280.15 \mathrm{~K}, \quad \varphi \equiv 0.40, \quad$ and $d_{s} \equiv 0.003 \mathrm{~m}$. The material-related dimensionless parameters remained constant.

\subsection{Effect of $\boldsymbol{R} \boldsymbol{e}_{s} / \boldsymbol{A} \boldsymbol{r}_{s}$ on $\boldsymbol{h}_{e} / \boldsymbol{d}_{s}$ and $\boldsymbol{c}_{d}$}

The dimensionless number $R e_{s} / A r_{s}$ was set by changing the inlet velocity of the mixture $v_{0}$ while all other dimensionless numbers were kept fixed at their base values. The considered range of $v_{0}$ was between the minimum fluidization velocity and the settling velocity of the particles, giving $0.07 \mathrm{~m} / \mathrm{s} \leq v_{0} \leq 0.26 \mathrm{~m} / \mathrm{s}$.

Fig. 6 a shows the computational results, which yielded the following correlation for $h_{e} / d_{s}$ as a function of $R e_{s} / A r_{s}$ :

$\frac{h_{e}}{d_{s}}=(3 e+6)\left(\frac{R e_{s}}{A r_{s}}\right)^{1.15}$.

Note that $b_{1}=1.15$. The effects of $R e_{s} / A r_{s}$ were mainly reflected in two aspects: (i) $R e_{s} / A r_{s}$ had an important effect on the concentration distribution of the solid phase and the expansion speed of the top height of particles. (ii) A larger $R e_{s}$ was beneficial to the convection heat transfer between the particles and water, thus speeding up hydrate dissociation. Meanwhile, the sparse particle distribution caused by the large $R e_{s} / A r_{s}$ also contributed to the heat transfer between the particles and water. Overall, the dissociation equilibrium height increased as $R e_{s} /$ $A r_{s}$ increased. Larger $R e_{s} / A r_{s}$ contributed to the upward movement of the particles, thus increasing $h_{e}$. Conversely, the larger $R e_{s}$ caused hydrate dissociation to occur faster, which had a tendency to reduce $h_{e}$. Thus, the effect of $R e_{s} / A r_{s}$ on $h_{e} / d_{s}$ resulted from the combination of the two opposite mechanisms, and the first mechanism was stronger than the second one, resulting in an approximately linear relationship between $h_{e} / d_{s}$ and $R e_{s} / A r_{s}$. This may also be a physical explanation for the deviation of individual data points from the fitted curve.

Fig. $6 \mathrm{~b}$ shows the following power-law correlation for $c_{d}$ and $R e_{s} / A r_{s}$ :

$c_{d}=(4 e-5)\left(\frac{R e_{s}}{A r_{s}}\right)^{-2.10}$.

Table 2

Properties of sand, hydrate, water, and methane (Li et al., 2019).

\begin{tabular}{|c|c|c|c|c|c|c|c|}
\hline Phase & & Density $\left(\mathrm{kg} / \mathrm{m}^{3}\right)$ & Diameter $(\mathrm{mm})$ & Viscosity (Pa.s) & Molar mass $(\mathrm{kg} / \mathrm{kmol})$ & Specific heat $(\mathrm{J} / \mathrm{kg} \cdot \mathrm{K})$ & Thermal conductivity $(\mathrm{W} / \mathrm{m} \cdot \mathrm{K})$ \\
\hline \multirow[t]{3}{*}{ Solid } & Sand $^{\text {a) }}$ & 2720 & 3 & - & 60 & 840 & 3 \\
\hline & Hydrate $^{\text {b) }}$ & 920 & & - & 119.5 & 2010 & 0.4 \\
\hline & Water $^{c)}$ & 1003.3 & & 0.001 & 18 & 4155 & 0.6 \\
\hline Liquid & Water ${ }^{c)}$ & 1003.3 & - & 0.001 & 18 & 4155 & 0.6 \\
\hline Gas & Methane $^{\mathrm{d})}$ & 79.68 & 0.3 & $1.4 \mathrm{e}-05$ & 16 & 3099 & 0.04 \\
\hline
\end{tabular}

a), b), c), d) Values are quoted from Bai et al. (2007), Sean et al. (2007), Wagner and Pruss (2002), and Friend et al. (1989), respectively. 

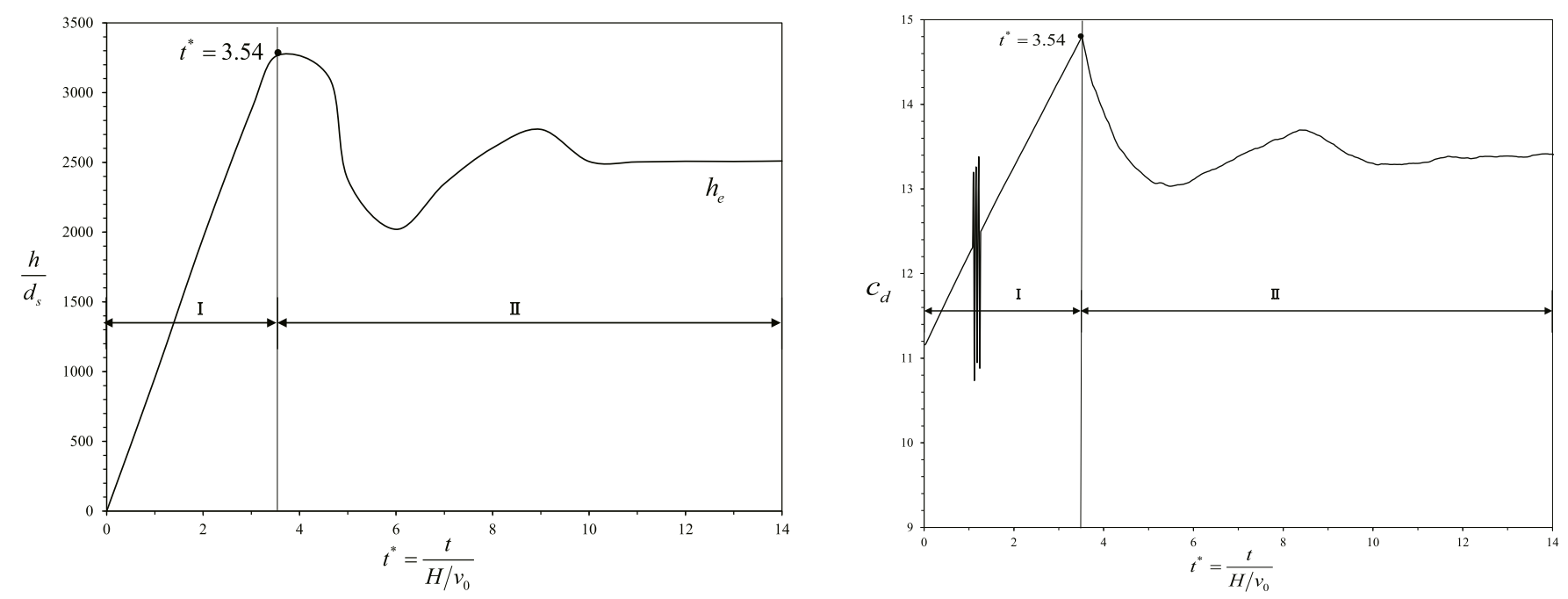

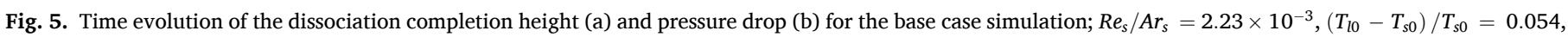
$\alpha_{s 0}=0.10, \varphi S_{H}=0.16$, and $d_{s} / D=0.030$.

Note that $b_{2}=-2.10$. This illustrates that the friction coefficient decreases with the increase of $\operatorname{Re}_{s} / A r_{s}$.

\subsection{Effect of $\left(\boldsymbol{T}_{l 0}-\boldsymbol{T}_{s 0}\right) / \boldsymbol{T}_{s 0}$ on $\boldsymbol{h}_{e} / \boldsymbol{d}_{s}$ and $\boldsymbol{c}_{d}$}

The temperature difference between particles and water was the main driving force of hydrate dissociation in this study, as shown in Eq. (3). Taking into account the temperature of the in-situ sediments, $T_{s 0}$ was assumed to be $280.15 \mathrm{~K}$. Because GH dissociation is an

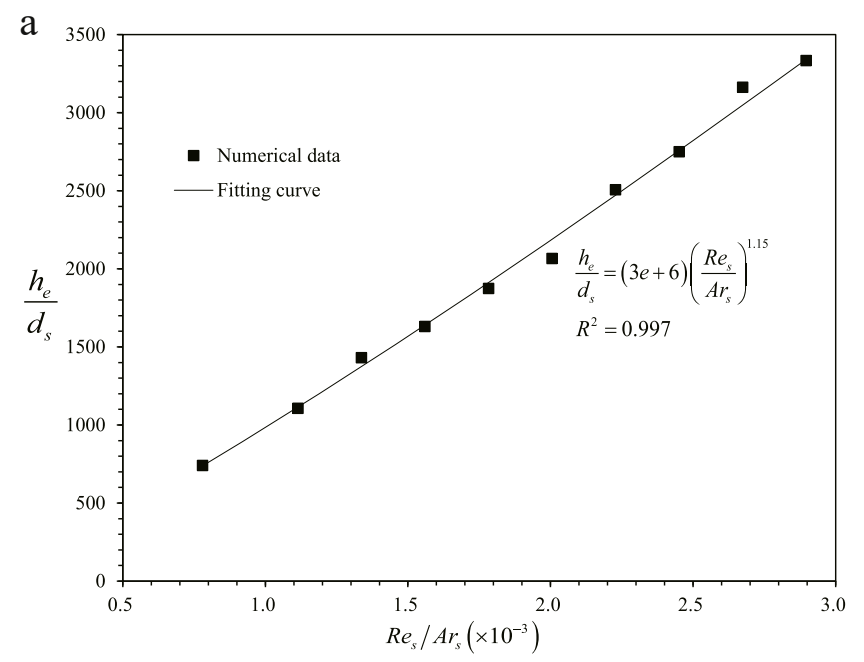

endothermic reaction, the injected water must provide the energy required for hydrate dissociation. Considering the limitations of engineering practice, the range of the water temperature was chosen as $295.15 \mathrm{~K} \leq T_{l 0} \leq 310.15 \mathrm{~K}$. Fig. 7a shows that the dissociation equilibrium height decreased with increasing water temperature as a higher temperature difference speeds up hydrate dissociation and thus decreases $h_{e}$. The correlation for $h_{e} / d_{s}$ as a function of $\left(T_{l 0}-T_{s 0}\right) / T_{s 0}$ is given in Fig. 7a:

$\mathrm{b}$

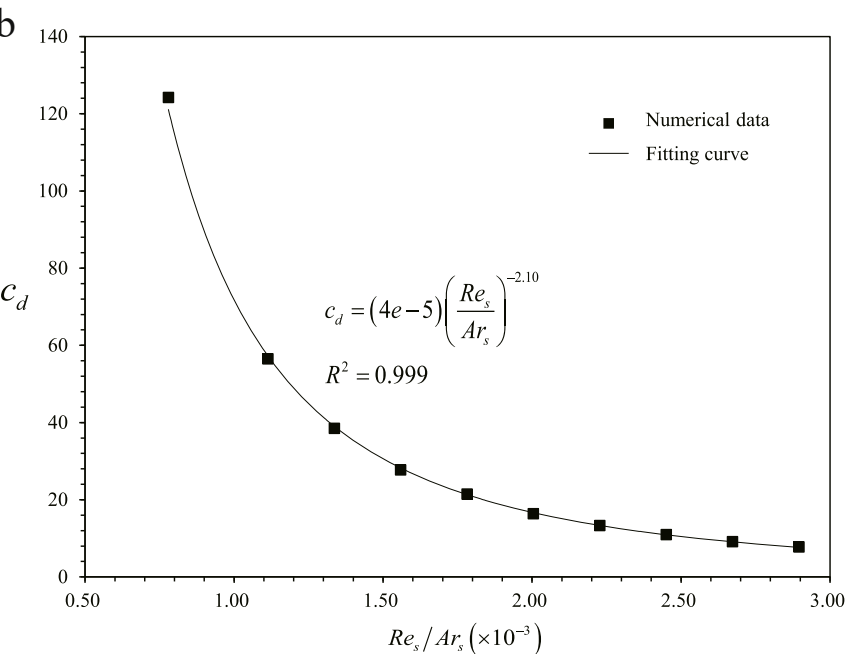

Fig. 6. The dependence of $h_{e} / d_{s}$ (a) and $c_{d}$ (b) on $R e_{s} / A r_{s}$ for $\left(T_{l 0}-T_{s 0}\right) / T_{s 0}=0.054, \alpha_{s 0}=0.10, \varphi S_{H}=0.16$, and $d_{s} / D=0.030$. 
Table 3

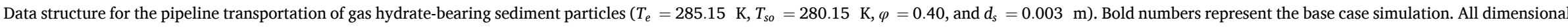
variables are given in SI units.

\begin{tabular}{|c|c|c|c|c|c|c|c|c|c|}
\hline$v_{0}$ & $T_{l 0}$ & $\alpha_{s 0}$ & $S_{H}$ & D & $R e_{s} / A r_{s}$ & $\begin{array}{l}\left(T_{10}-\right. \\
\left.T_{s 0}\right) / T_{s 0}\end{array}$ & $\varphi S_{H}$ & $d_{s} / D$ & $h_{e} / d_{s}$ \\
\hline $\begin{array}{l}0.07,0.10,0.12 \\
0.14,0.16,0.18 \\
\mathbf{0 . 2 0}, 0.22,0.24 \\
0.26\end{array}$ & 295.15 & 0.10 & 0.40 & 0.100 & $\begin{array}{l}7.80 \times 10^{-4}, 1.11 \times 10^{-3}, 1.34 \times 10^{-3}, \\
1.56 \times 10^{-3}, 1.78 \times 10^{-3}, 2.01 \times 10^{-3}, \\
2.23 \times 10^{-3}, 2.45 \times 10^{-3}, 2.67 \times 10^{-3} \\
2.90 \times 10^{-3}\end{array}$ & 0.054 & 0.16 & 0.030 & $\begin{array}{l}740.00,1,106.67,1,430.00, \\
1,630.00,1,873.33,2,066.67, \\
2,506.67,2,750.00,3,163.33, \\
3,333.33\end{array}$ \\
\hline 0.20 & $\begin{array}{l}297.15,299.15 \\
300.15,302.15 \\
304.15,305.15 \\
307.15,310.15\end{array}$ & 0.10 & 0.40 & 0.100 & $2.23 \times 10^{-3}$ & $\begin{array}{l}0.061, \\
0.068, \\
0.071, \\
0.079, \\
0.086, \\
0.089, \\
0.096, \\
0.107\end{array}$ & 0.16 & 0.030 & $\begin{array}{l}1,906.67,1,460.00,1,326.67 \\
1,080.00,870.00,766.67 \\
620.00,436.67\end{array}$ \\
\hline 0.20 & 295.15 & $\begin{array}{l}0.04,0.05 \\
0.06,0.07 \\
0.08,0.09 \\
0.11,0.12\end{array}$ & 0.40 & 0.100 & $2.23 \times 10^{-3}$ & 0.054 & 0.16 & 0.030 & $\begin{array}{l}1,026.67,1,293.33,1,483.33 \\
1,776.67,2,016.67,2,196.67 \\
2,506.67,2,746.67\end{array}$ \\
\hline 0.20 & 295.15 & 0.10 & $\begin{array}{l}0.10,0.15 \\
0.20,0.25 \\
0.30,0.35 \\
0.45,0.50\end{array}$ & 0.100 & $2.23 \times 10^{-3}$ & 0.054 & $\begin{array}{l}0.04,0.06 \\
0.08,0.10 \\
0.12,0.14 \\
0.18,0.20\end{array}$ & 0.030 & $\begin{array}{l}1,346.67,1,606.67,1,793.33 \\
1,990.00,2,063.33,2,320.00 \\
2,643.33,2,816.67\end{array}$ \\
\hline 0.20 & 295.15 & 0.10 & 0.40 & $\begin{array}{l}0.300,0.200 \\
0.176,0.150 \\
0.120,0.086 \\
0.075,0.067\end{array}$ & $2.23 \times 10^{-3}$ & 0.054 & 0.16 & $\begin{array}{l}0.010,0.015 \\
0.017,0.020 \\
0.025,0.035 \\
0.040,0.045\end{array}$ & $\begin{array}{l}2,576.67,2,446.67,2,563.33 \\
2,610.00,2,620.00,2,526.67 \\
2,510.00,2,540.00\end{array}$ \\
\hline
\end{tabular}




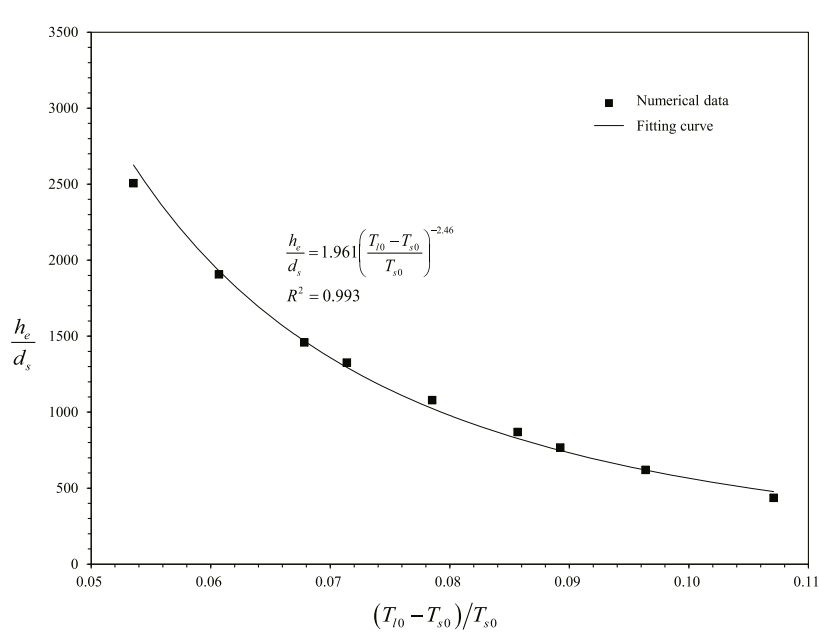

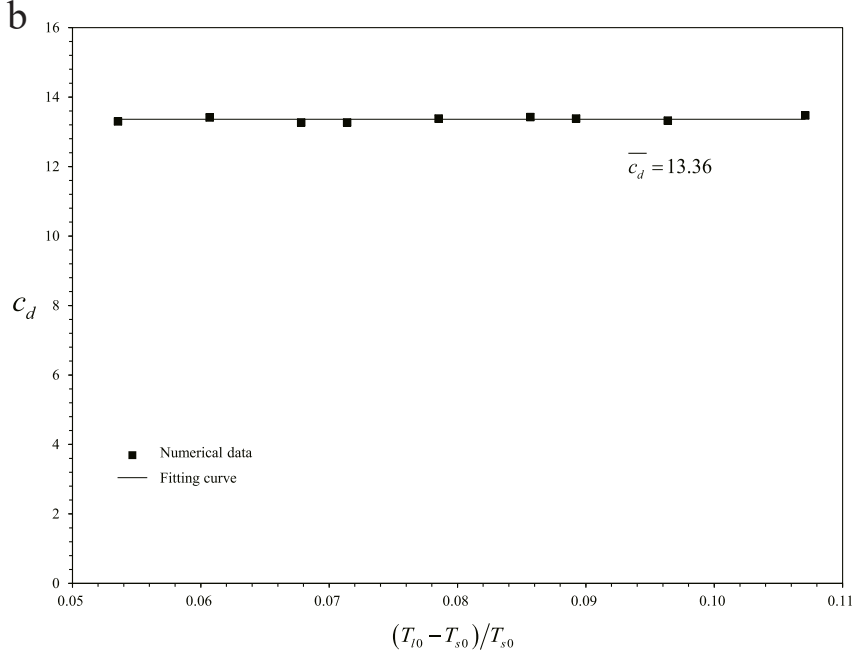

Fig. 7. The dependence of $h_{e} / d_{s}$ (a) and $c_{d}$ (b) on $\left(T_{l 0}-T_{s o}\right) / T_{s 0}$ for $R e_{s} / A r_{s}=2.23 \times 10^{-3}, \alpha_{s 0}=0.10, \varphi S_{H}=0.16$, and $d_{s} / D=0.030$. 

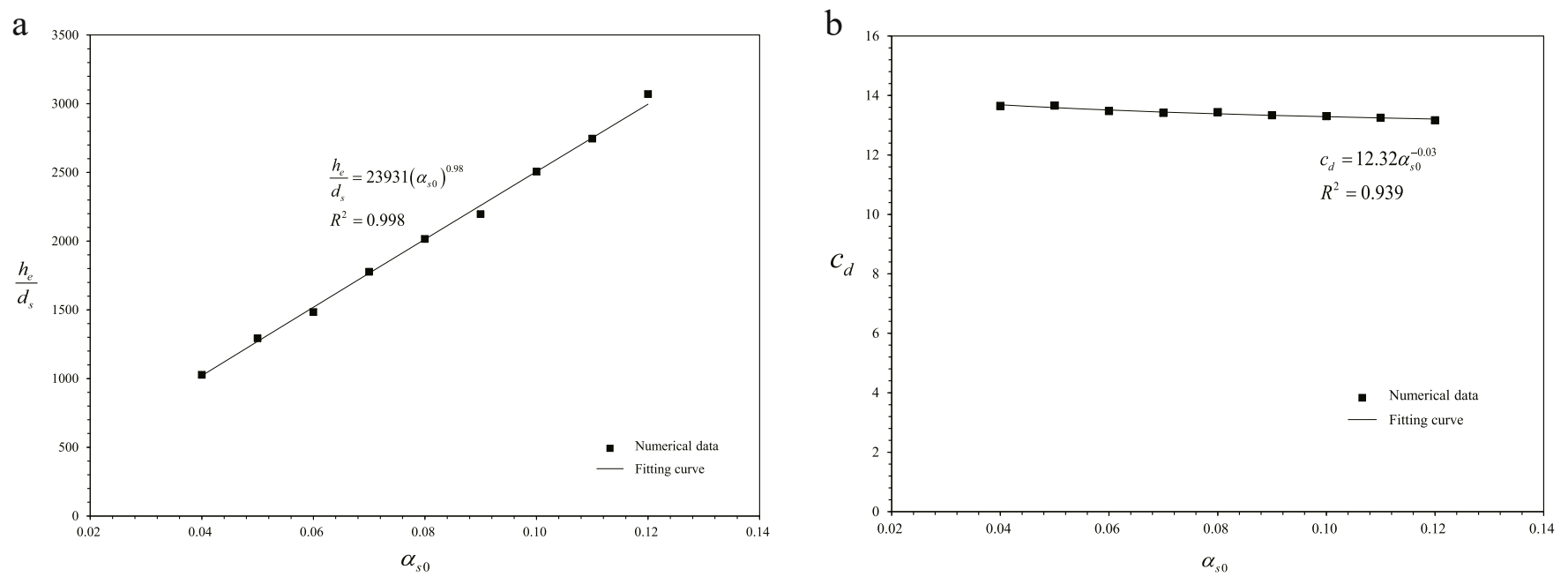

Fig. 8. The dependence of $h_{e} / d_{s}$ (a) and $c_{d}$ (b) on $\alpha_{s 0}$ for $R e_{s} / A r_{s}=2.23 \times 10^{-3},\left(T_{l 0}-T_{s 0}\right) / T_{s 0}=0.054, \varphi S_{H}=0.16$, and $d_{s} / D=0.030$. 
a

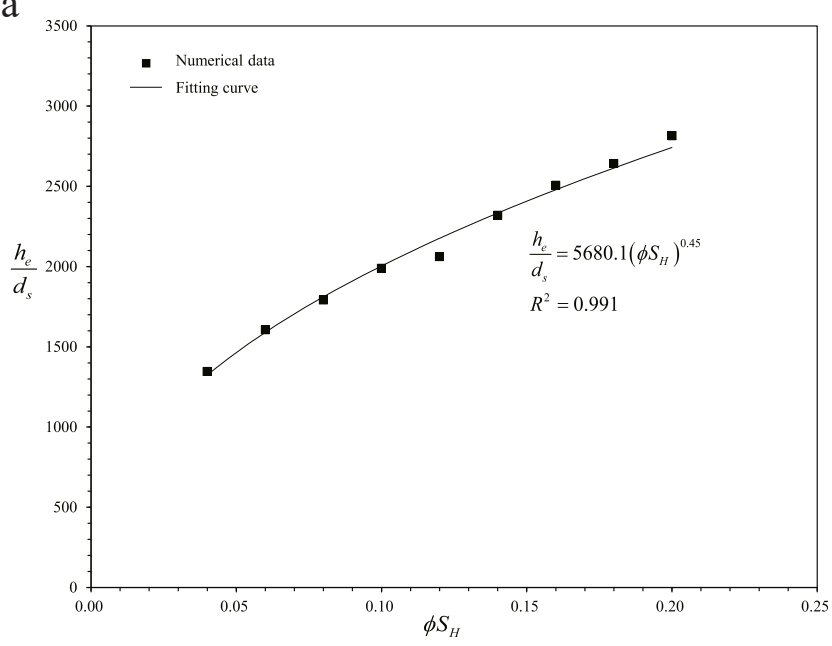

b

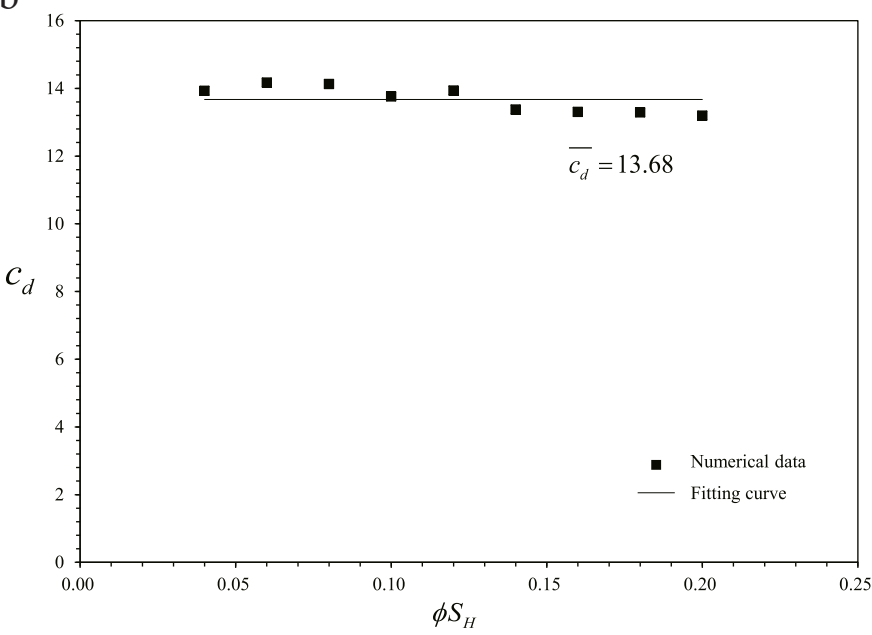

Fig. 9. The dependence of $h_{e} / d_{s}$ (a) and $c_{d}$ (b) on $\varphi S_{H}$ for $R e_{s} / A r_{s}=2.23 \times 10^{-3},\left(T_{l 0}-T_{s 0}\right) / T_{s 0}=0.054, \alpha_{s 0}=0.10$, and $d_{s} / D=0.030$.

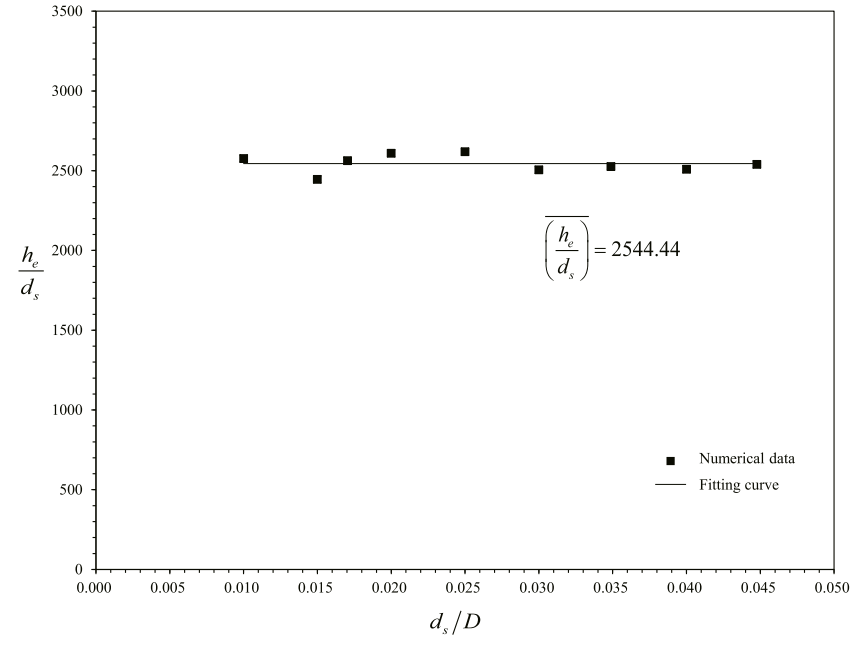

Fig. 10. The dependence of $h_{e} / d_{s}$ on $d_{s} / D$ for $R e_{s} / A r_{s}=2.23 \times 10^{-3},\left(T_{l 0}-\right.$ $\left.T_{s 0}\right) / T_{s 0}=0.054, \alpha_{s 0}=0.10$, and $\varphi S_{H}=0.16$.

$\frac{h_{e}}{d_{s}}=1.961\left(\frac{T_{l 0}-T_{s 0}}{T_{s 0}}\right)^{-2.46}$.

Note that $c_{1}=-2.46$.

Fig. $7 \mathrm{~b}$ shows that $c_{d}$ was almost independent of the temperature difference. The deviation between all data points and the mean value of $c_{d}(=13.36)$ was no more than $0.9 \%$. Therefore, $\left(T_{l 0}-T_{s o}\right) / T_{s 0}$ can be considered to have no effect on $c_{d}$, giving $c_{2}=0$.

\subsection{Effect of $\boldsymbol{\alpha}_{s 0}$ on $\boldsymbol{h}_{\boldsymbol{e}} / \boldsymbol{d}_{s}$ and $\boldsymbol{c}_{\boldsymbol{d}}$}

The initial solid volume fraction $\alpha_{s 0}$ is a very important dimensionless number for transportation of GHBS particles in the pipe. There are two mechanisms for the influence of $\alpha_{s 0}$ on $h_{e} / d_{s}$ :

(i) A larger $\alpha_{s 0}$ indicates an increase in the concentration of hydrate in the pipe, which leads to an increase in the hydrate dissociation time. There is a tendency for $h_{e}$ to increase.

(ii) The increase of $\alpha_{s 0}$ is equivalent to increasing the amount of particles in the pipe, which thereby affects the transport efficiency of the solid particles in the pipe and prolongs the dissociation time. There is also a tendency for $h_{e}$ to increase.

Under the combined effect of these two mechanisms, $h_{e} / d_{s}$ increases with the increase of $\alpha_{s 0}$. In general, the correlation between $h_{e} / d_{s}$ and $\alpha_{s 0}$ shown in Fig. 8a is expressed as:

$\frac{h_{e}}{d_{s}}=23931\left(\alpha_{s 0}\right)^{0.98}$.

Note that $d_{1}=0.98$.

Fig. 8b shows that $c_{d}$ decreased slowly as $\alpha_{s 0}$ increased, indicating that $\alpha_{s 0}$ had a slight effect on $c_{d}$. The correlation for $c_{d}$ as a function of $\alpha_{s 0}$ is expressed as:

$c_{d}=12.32 \alpha_{s 0}^{-0.03}$.

Note that $d_{2}=-0.03$.

\subsection{Effect of $\varphi \boldsymbol{S}_{\boldsymbol{H}}$ on $\boldsymbol{h}_{\boldsymbol{e}} / \boldsymbol{d}_{\boldsymbol{s}}$ and $\boldsymbol{c}_{\boldsymbol{d}}$}

In the following simulations, the $\mathrm{GH}$ saturation $S_{H}$ was set as $0.10 \leq S_{H} \leq 0.50$. Therefore, $0.04 \leq \varphi S_{H} \leq 0.20$. As shown in Fig. 9a, the variation of $h_{e} / d_{s}$ for $\varphi S_{H}$ can be described as:

$\frac{h_{e}}{d_{s}}=5680.1\left(\varphi S_{H}\right)^{0.45}$.

Note that $e_{1}=0.45$. According to the definition of $\varphi S_{H}$, a larger $\varphi S_{H}$ means more hydrate in particles, which results in an increase in the dissociation time. Moreover, a larger $\varphi S_{H}$ means more gas production in the pipe, which then increases the expansion height of the

Table 4

The curve-fitting correlations of $h_{e} / d_{s}$ and $c_{d}$ for various dimensionless numbers.

\begin{tabular}{|c|c|c|c|c|}
\hline Dependent variable & $R e_{s} / A r_{s}$ & $\left(T_{l 0}-T_{s 0}\right) / T_{s 0}$ & $\alpha_{s 0}$ & $\varphi S_{H}$ \\
\hline $\begin{array}{l}\text { Dissociation equilibrium height } h_{e} / d_{s} \\
\text { Friction coefficient } c_{d}\end{array}$ & $\begin{array}{l}\frac{h_{e}}{d_{s}}=(3 e+6)\left(\frac{R e_{s}}{A r_{s}}\right)^{1.15} \\
c_{d}=(4 e-5)\left(\frac{R e_{s}}{A r_{s}}\right)^{-2.10}\end{array}$ & $\frac{h_{e}}{d_{s}}=1.961\left(\frac{T_{l 0}-T_{s 0}}{T_{s 0}}\right)^{-2.46}$ & $\begin{array}{l}\frac{h_{e}}{d_{s}}=23931\left(\alpha_{s 0}\right)^{0.98} \\
c_{d}=12.32 \alpha_{s 0}^{-0.03}\end{array}$ & $\frac{h_{e}}{d_{s}}=5680.1\left(\varphi S_{H}\right)^{0.45}$ \\
\hline
\end{tabular}




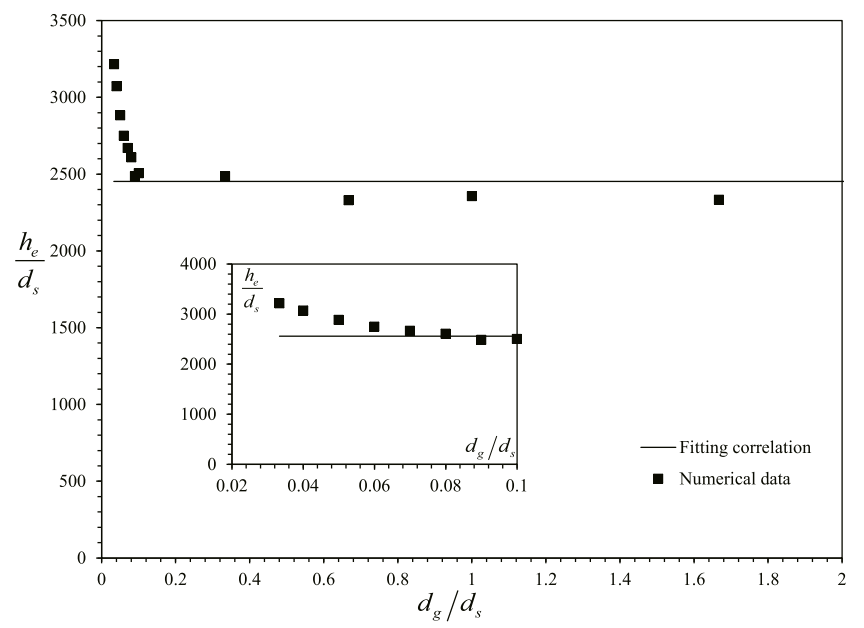

Fig. 11. The values predicted of $h_{e} / d_{s}$ by Eq. (23) versus the simulation data for different $d_{g} / d_{s}$ cases. Other settings are the same as the base case.

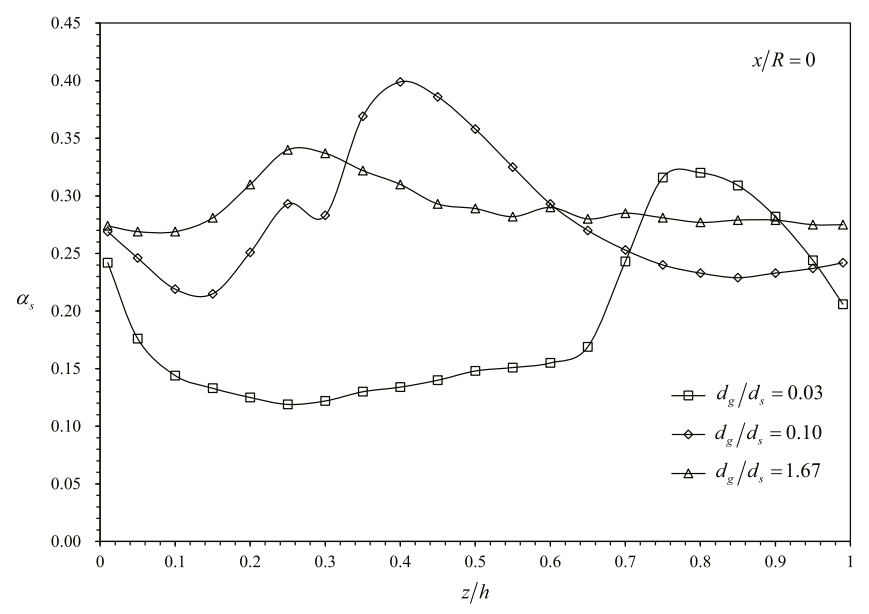

Fig. 12. Time-averaged solid volume fraction versus the length of the pipe for three different $d_{g} / d_{s}$ cases under stable state conditions. When the steady state was reached, data were recorded for an additional $100 \mathrm{~s}$ to obtain the timeaveraged quantities.

gas-liquid-solid flow. These two mechanisms eventually lead to an increase in $h_{e} / d_{s}$ with increasing $\varphi S_{H}$.

Fig. $9 \mathrm{~b}$ shows that the variance of $\varphi S_{H}$ had an effect on $c_{d}$ similar to that of $\left(T_{l 0}-T_{s 0}\right) / T_{s 0}$. The deviation between all data points and the mean value of $c_{d}(=13.68)$ was no more than $3.7 \%$. Therefore, $\varphi S_{H}$ is also considered to have no effect on $c_{d}$, giving $e_{2}=0$.

According to Eq. (4), the density of GHBS particles $\rho_{s}$ decreases as the saturation $S_{H}$ increases, which leads to the decrease of $A r_{s}$. The dimensionless numbers $\varphi S_{H}$ and $A r_{s}$ are not independent of each other. In the range of $0.04 \leq \varphi S_{H} \leq 0.20$, leading to $2.69 \times 10^{5} \leq A r_{s} \leq 2.73 \times 10^{5}$, a five-fold increase in $\varphi S_{H}$ decreases the value of $A r_{s}$ by only about $1.5 \%$. Therefore, for simplicity, only the effect of $\varphi S_{H}$ was considered in this study.

\subsection{Effect of $\boldsymbol{d}_{s} / \mathrm{D}$ on $\boldsymbol{h}_{\boldsymbol{e}} / \boldsymbol{d}_{s}$}

The ratio $d_{s} / D$ was set by changing the pipe diameter $D$. The variation range of $d_{s} / D$ was $0.010 \leq d_{s} / D \leq 0.045$. The dependence of $h_{e} / d_{s}$ on $d_{s} / D$ is plotted in Fig. $10 ; h_{e} / d_{s}$ did not change significantly with $d_{s} / D$. The deviation between all data points and the mean value of $h_{e} / d_{s}(=$

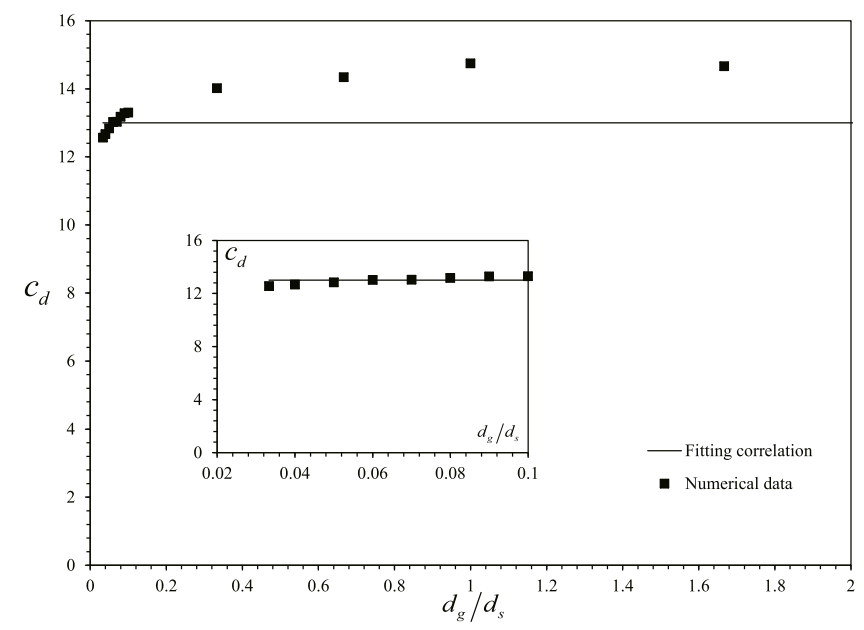

Fig. 13. The values of $c_{d}$ predicted by Eq. (24) versus the simulation data for different $d_{g} / d_{s}$ cases. Other settings are the same as the base case.

$2,544.44)$ was no more than $4 \%$. Therefore, $d_{s} / D$ can be considered to have had no effect on $h_{e} / d_{s}$, giving $f_{1}=0$. This result indicates that $h_{e} / d_{s}$ is not dependent on the size of the pipe; the present result concerning the correlation of $h_{e} / d_{s}$ may be extended for the engineering scale.

\subsection{Final states and applicability of fitting correlations}

The variances of $h_{e} / d_{s}$ and $c_{d}$ for various dimensionless numbers are summarized in Figs. 6-10. It is clear that $h_{e} / d_{s}$ increased with larger $R e_{s} / A r_{s}, \alpha_{s 0}$, and $\varphi S_{H}$, and with smaller $\left(T_{l 0}-T_{s 0}\right) / T_{s 0}$. The variance of $d_{s} / D$ had almost no influence on the value of $h_{e} / d_{s}$. In addition, $c_{d}$ was only a function of $R e_{s} / A r_{s}$ and $\alpha_{s 0}$, and the value of $c_{d}$ increased with decrease in $R e_{s} / A r_{s}$ and $\alpha_{s 0}$. The curve-fitting correlations of $h_{e} / d_{s}$ and $c_{d}$ for various dimensionless numbers are summarized in Table 4.

Thus, the power-law correlations between $h_{e} / d_{s}, c_{d}$, and the dimensionless numbers can be expressed as follows:

$\left.\begin{array}{l}\frac{h_{e}}{d_{s}}=a_{1}\left(\frac{R e_{s}}{A r_{s}}\right)^{b_{1}}\left(\frac{T_{l 0}-T_{s 0}}{T_{s 0}}\right)^{c_{1}}\left(\alpha_{s 0}\right)^{d_{1}}\left(\varphi S_{H}\right)^{e_{1}} \\ c_{d}=a_{2}\left(\frac{R e_{s}}{A r_{s}}\right)^{b_{2}}\left(\alpha_{s 0}\right)^{d_{2}}\end{array}\right\}$.

The data obtained from the simulation were then fitted into the most suitable correlations through the least squares method. The average values of the pre-factors $a_{1}$ and $a_{2}$ were also obtained from the curve fit for all data points. The final form of the power-law correlations for $h_{e} / d_{s}$ and $c_{d}$ are:

$$
\begin{aligned}
\frac{h_{e}}{d_{s}} & =9.17 \times 10^{4}\left(\frac{R e_{s}}{A r_{s}}\right)^{1.17}\left(\frac{T_{l 0}-T_{s 0}}{T_{s 0}}\right)^{-2.28}\left(\alpha_{s 0}\right)^{0.99}\left(\varphi S_{H}\right)^{0.47}, \\
c_{d} & =2.34 \times 10^{-5}\left(\frac{R e_{s}}{A r_{s}}\right)^{-2.14}\left(\alpha_{s 0}\right)^{-0.06} .
\end{aligned}
$$

Note that $a_{1}=9.17 \times 10^{4}$ and $a_{2}=2.34 \times 10^{-5}$. The coefficients of determination $R^{2}$ of Eqs. 23 and 24 are 0.996 and 0.999 , respectively. The exponents $b_{1}, c_{1}, d_{1}, e_{1}, b_{2}$, and $d_{2}$ changed slightly from the original values for better agreement between the data and the correlations. In this study, the data from which the correlations were derived had the following ranges of parameters: $7.80 \times 10^{-4} \leq R e_{s} / A r_{s} \leq 2.90 \times 10^{-3}$, $0.054 \leq\left(T_{l 0}-T_{s 0}\right) / T_{s 0} \leq 0.107,0.04 \leq \alpha_{s 0} \leq 0.12,0.04 \leq \varphi S_{H} \leq 0.20$ and $0.010 \leq d_{s} / D \leq 0.045$.

The above fitting correlations do not take into account the effect of pressure change on the hydrate dissociation rate. When the dissociation height is large enough that the pressure change has a significant effect 
on the dissociation rate, the errors of the fitting correlations become larger.

During GH dissociation, the bubbles gradually move away from the cracks of the sediment particles. The initial bubble size is important in the hydrodynamic characterization of the gas-liquid-solid flow system. Additionally, bubble size always changes because of the coalescence and breakup of bubbles. Prediction of the bubble size distribution in the gas-liquid-solid flow is quite complex. Therefore, a constant mean bubble size was assumed in the present study; Eq. (23) and Eq. (24) were obtained based on a constant bubble diameter, $d_{g} / d_{s}=0.10$. To test the applicability of the correlations for different bubble sizes, simulations with various bubble diameters were carried out to explore the effect of $d_{g} / d_{s}$ on $h_{e} / d_{s}$ and $c_{d}$. The variation range of $d_{g}$ was $0.1 \mathrm{~mm} \leq d_{g} \leq$ $5 \mathrm{~mm}$; thus, $0.03 \leq d_{g} / d_{s} \leq 1.67$. Other setting were the same as the base case.

Fig. 11 shows a comparison between the values of $h_{e} / d_{s}$ predicted by Eq. (23) and simulation values for different $d_{g} / d_{s}$ cases. The equilibrium height became smaller with the increase of the bubble diameter. The following is a possible physical explanation. The increase of bubble diameter could lead to the increase in inertia and buoyancy of bubbles, resulting in the increase of $R e_{g}$ and $A r_{g}$, consequently increasing the velocity of the bubbles. The change in bubble rise velocity further resulted in the variation of solid concentration. Fig. 12 shows the distribution of the volume fraction of solid particles along the pipe at stable states. The solid volume fraction was more homogeneous along the pipe when the bubble diameter was large. This finding indicates that the larger bubble size provided a better mixing in the axial direction for the solid particles. In addition, the solid concentration increased with the increase in bubble size. This was mainly because the increase in bubble diameter reduced the gas concentration. For the same number of particles, a higher average concentration of particles in the pipe meant that the distribution height was smaller. Thus, a larger $d_{g} / d_{s}$ was associated with a trend of decreasing $h_{e} / d_{s}$. Fig. 11 also shows that the values predicted by Eq. (23) are in good agreement with the simulation data for $0.06 \leq d_{g} / d_{s} \leq 1.67$, with an error of less than $10 \%$. Eq. (23) can be considered applicable for $0.06 \leq d_{g} / d_{s} \leq 1.67$.

Based on the above analysis, the assumption that the effects of $R e_{g}$ and $A r_{g}$ on the bubble movement can be characterized by $d_{g} / d_{s}$ is reasonable.

Fig. 13 shows a comparison between the values of $c_{d}$ predicted by Eq. (24) and simulation values for different $d_{g} / d_{s}$ cases. With the increase of bubble diameter, $c_{d}$ tended to increase slowly. Equation (24) can be used to predict changes in $c_{d}$ in the case of $0.03 \leq d_{g} / d_{s} \leq 0.67$; the two results have error of less than $10 \%$.

Generally, the errors of Eqs. (23) and (24) may derive from the following aspects:

(i) The model of gas-liquid-solid three-phase flow coupled with heat and mass transfer is very complex; the choice of empirical closed equations for the solid phase and the equations for the interphase momentum and heat exchange may cause errors in the numerical simulation results.

(ii) The effect of the pressure change on the hydrate dissociation rate is not considered. The hydrate dissociation kinetic model plays a decisive role in the formation of multiphase flow. Therefore, the more precise kinetic parameters for $\mathrm{GH}$ dissociation under multiphase flow help to determine pipe flow behavior more accurately.

(iii) The power-law correlations are obtained by fitting a limited range of data, and the material properties are assumed to be unchanged during upward flow of the mixture of warmer seawater and GHBS particles.

\section{The analytical expression of the dissociation equilibrium height}

In the above sections, the curve-fitting correlation for $h_{e} / d_{s}$ was obtained. The fitting correlation does not take into account the effect of pressure change on the hydrate dissociation rate, and its application scope is limited. In addition, because the multiphase flow transport with GH dissociation is a newly presented problem in the area of exploitation of $\mathrm{GH}$, to the best of our knowledge, no experimental studies on dissociation equilibrium height have been reported to data. To verify the correctness of the power-law correlation and provide better support for future engineering practice, for this section, the analytical expression of $h_{e} / d_{s}$ was obtained by decoupling hydrate dissociation and solid-liquid flow, and its applicability was evaluated.

\subsection{Decoupling of hydrate dissociation and solid-liquid flow}

If hydrate dissociation is not considered, only solid-liquid two-phase flow exists in the pipe. The solid particles can be considered as homogeneously distributed in the flow field when the system is stable. Therefore, the volume fraction of the solid phase $\alpha_{s}$ is constant. The value of $\alpha_{s}$ can be given by the well-known Richardson-Zaki correlation (Richardson and Zaki, 1954):

$\frac{v_{0}}{v_{t}}=\left(1-\alpha_{s}\right)^{n}$,

where $v_{t}$ is the terminal velocity of solid particles, and $n$ is an expansion index. A correlation for $v_{t}$ developed by Haider and Levenspiel (1989) is given by:

$$
\left.\begin{array}{l}
v_{*}=\left[\frac{18}{d_{*}^{2}}+\frac{0.5909}{d_{*}^{0.5}}\right]^{-1} \\
v_{*}=v_{t}\left[\frac{\rho_{l}^{2}}{g \mu_{l}\left(\rho_{s}-\rho_{l}\right)}\right]^{\frac{1}{3}} \\
d_{*}=d_{s}\left[\frac{g \rho_{l}\left(\rho_{s}-\rho_{l}\right)}{\mu_{l}^{2}}\right]^{\frac{1}{3}}
\end{array}\right\} .
$$

The density of solid particles $\rho_{s}$ continues to increase during GH dissociation. In calculating $v_{t}, \rho_{s}$ is given by the mean value of the initial density of particles and the density after complete dissociation; $\rho_{s}$ is expressed as:

$\rho_{s}=\frac{1}{2}\left[(1-\varphi) \frac{2-\varphi S_{H}}{1-\varphi S_{H}} \rho_{\text {sand }}+\varphi S_{H} \rho_{\mathrm{GH}}+\varphi\left(1-S_{H}\right) \frac{2-\varphi S_{H}}{1-\varphi S_{H}} \rho_{\text {water }}\right]$.

The order $n$ is calculated from the correlation of Khan and Richardson (1989):

$\frac{4.8-n}{n-2.4}=0.043 A r_{s}^{0.57}\left[1-1.24\left(d_{s} / D\right)^{0.27}\right]$.

The solid-liquid mixture is injected into the pipe at a certain velocity $v_{0}$, and after a period of time $t$, the top height of the particles $h$ can be expressed as:

$h=\frac{4 M}{\pi D^{2} \rho_{s} \alpha_{s}}$,

where $M$ is the total mass of particles entering the pipe, $M=$ $(\pi / 4) D^{2} v_{0} t \alpha_{s 0} \rho_{s}$. Substituting $M=(\pi / 4) D^{2} v_{0} t \alpha_{s 0} \rho_{s}$ in (29) yields:

$h=\frac{v_{0} \alpha_{s 0}}{\alpha_{s}} t$.

The order of magnitude of the expansion velocity of the solid particles can be estimated by $v_{0} \alpha_{s 0} / \alpha_{s}$. If the characteristic length $=d_{s}$, the characteristic time of solid particles expansion is about: 


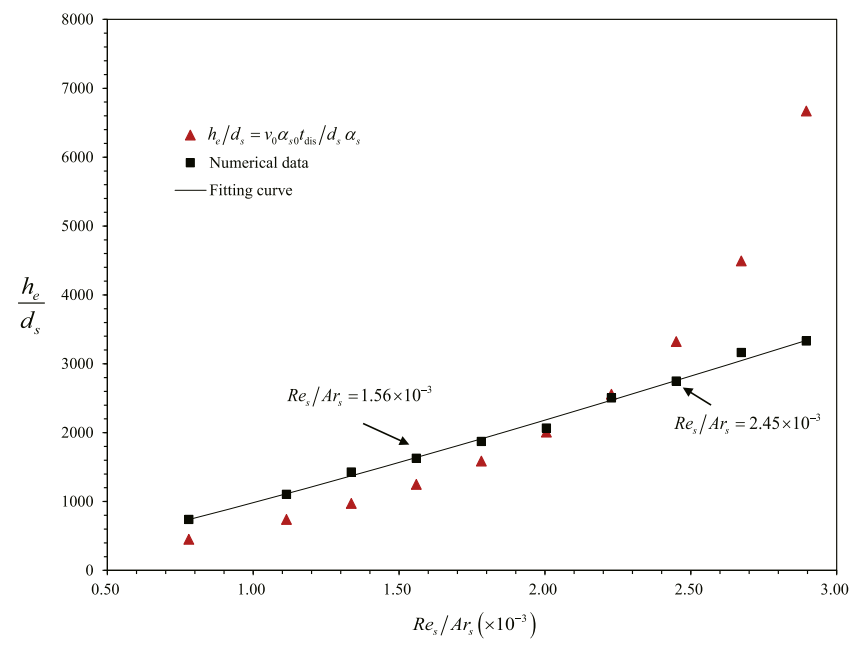

Fig. 14. The values of $h_{e} / d_{s}$ predicted by Eq. (36) versus the numerical simulated values for various values $R e_{s} / A r_{s}$ listed in Table 3 .

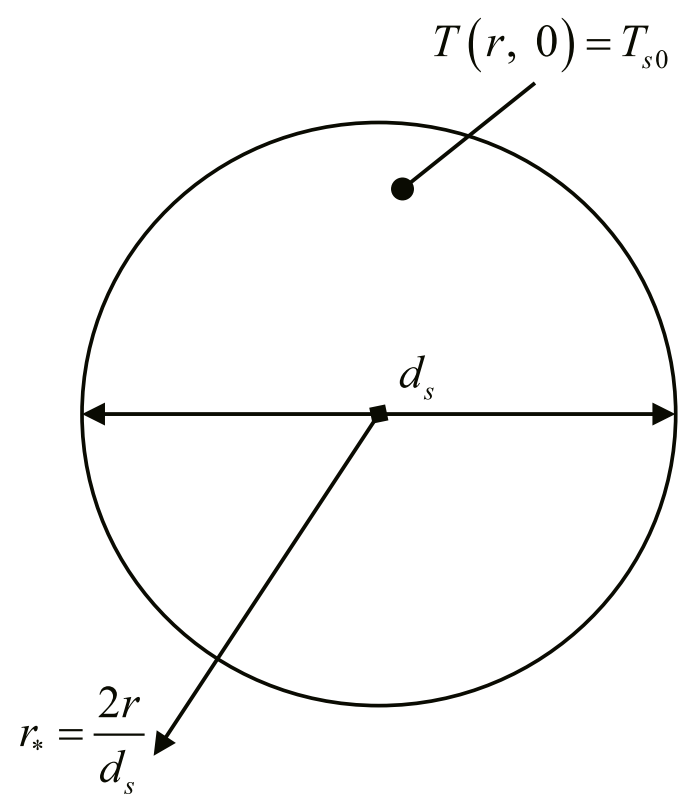

$T_{10}, \Delta v$

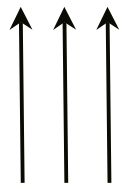

Fig. 15. A solid particle with an initial uniform temperature subjected to convection heat transfer boundary conditions.

$t_{\text {exp }}=\frac{d_{s} \alpha_{s}}{v_{0} \alpha_{s 0}}$.

If $t_{\text {exp }}$, which represents upward flow of solid particles, has the same order of magnitude as the time representing GHBS dissociation $t_{\mathrm{dis}}$, the effects of hydrate dissociation and solid-liquid flow are coupled and should be considered together. To estimate $t_{\text {dis }}$, it is assumed in Eq. (3) that $T_{s}=T_{l 0}$ and does not change with time, which means the heat transfer process between the solid particles and water is rapid, and the temperature of the particles quickly rises from the initial value $T_{s 0}$ to the maximum value $T_{10}$. The validity of this assumption is discussed in Section 5.2. Therefore, $k$ is a constant in Eq. (2). The time integral over Eq. (2) can reveal the relation between the dissociation time and hydrate concentration:

$t=\frac{1}{k} \cdot \frac{1}{1-\eta}\left(C_{0}^{1-\eta}-C_{\mathrm{GH}}^{1-\eta}\right)$,

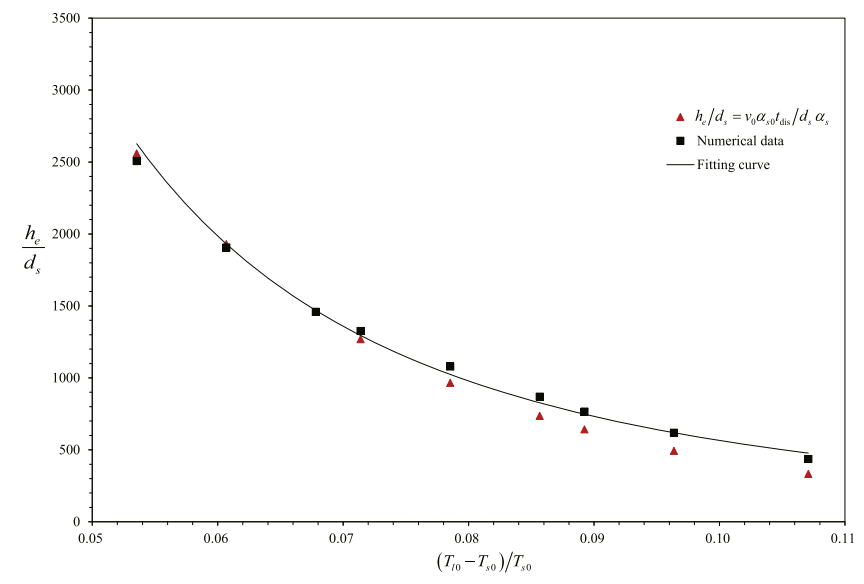

Fig. 16. The values of $h_{e} / d_{s}$ predicted by Eq. (36) versus the numerical simulated values for various values of $\left(T_{l 0}-T_{s 0}\right) / T_{s 0}$ listed in Table 3 .

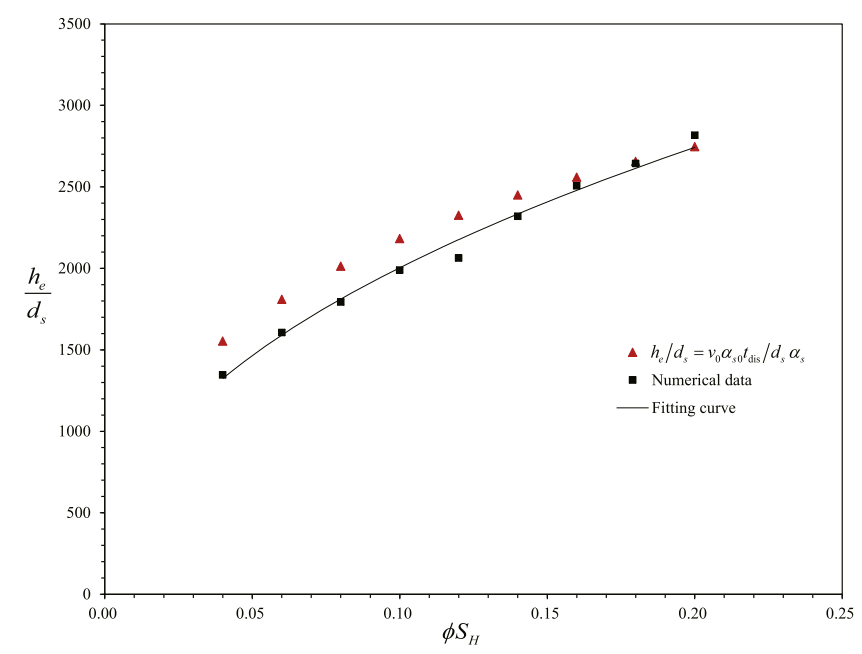

Fig. 17. The values of $h_{e} / d_{s}$ predicted by Eq. (36) versus the numerical simulated values for various values of $\varphi S_{H}$ listed in Table 3.

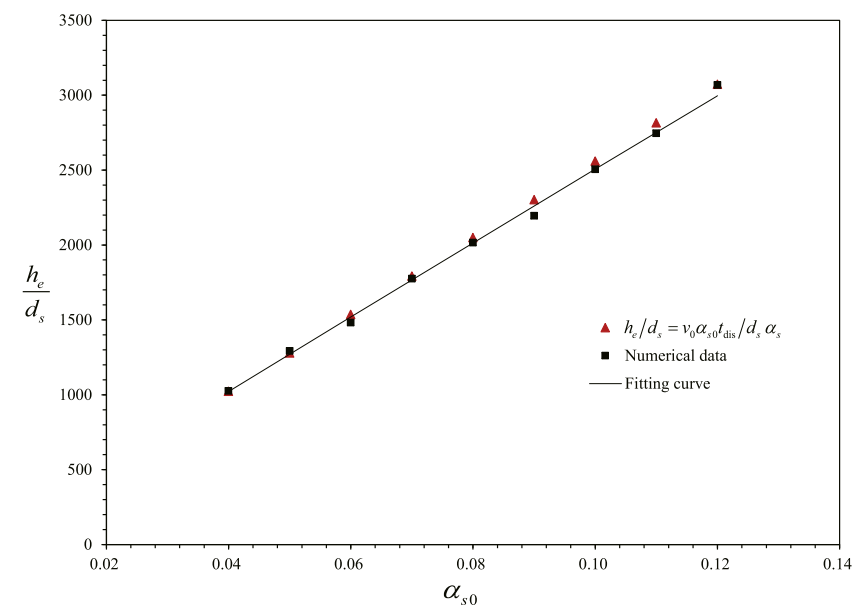

Fig. 18. The values of $h_{e} / d_{s}$ predicted by Eq. (36) versus the numerical simulated values for various values of $\alpha_{s 0}$ listed in Table 3. 


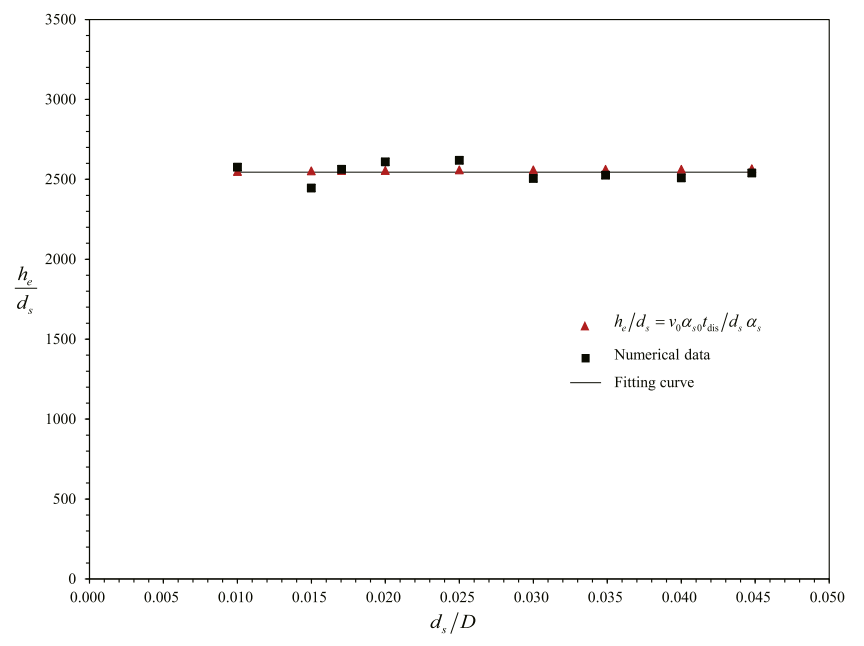

Fig. 19. The values of $h_{e} / d_{s}$ predicted by Eq. (36) versus the numerical simulated values for various values of $d_{s} / D$ listed in Table 3 .

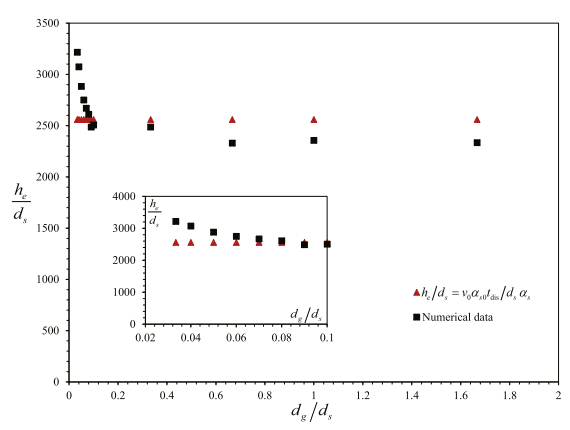

Fig. 20. The values of $h_{e} / d_{s}$ predicted by Eq. (36) versus the simulation data for different $d_{g} / d_{s}$ cases. Other settings are the same as the base case.

where $C_{0}$ is the initial hydrate concentration, $C_{0}=Y_{\mathrm{GH}} \rho_{s} \alpha_{s} / M_{\mathrm{GH}}$, and $Y_{\mathrm{GH}}$ is the mass fraction of hydrate in particles. When $C_{\mathrm{GH}}=0$,

$t_{\mathrm{dis}}=\frac{1}{k} \cdot \frac{1}{1-\eta} C_{0}^{1-\eta}$.

In general, it is easy to see that $t_{\mathrm{exp}} \ll t_{\text {dis }}$. For the base case, $t_{\mathrm{exp}}=$ $0.02 \mathrm{~s}$ and $t_{\mathrm{dis}}=50.89 \mathrm{~s}$. The ratio of the two characteristic times is:

$\frac{t_{\text {exp }}}{t_{\text {dis }}}=3.93 \times 10^{-3}$.

In this case, the two characteristic times differ by nearly three orders of magnitude. Hydrate dissociation is much slower than upward flow of solid particles; therefore, hydrate dissociation and solid-liquid flow can be viewed as being decoupled. First, the effect of solid-liquid flow on hydrate dissociation is not considered, and the time required for the complete dissociation of hydrate, $t_{\mathrm{dis}}$, is calculated based on Eq. (33). Then, the height of the upward flow of solid particles with the range of $t_{\text {dis }}$ is calculated based on Eq. (30). The calculated height is the dissociation equilibrium height $h_{e}$ :

$h_{e}=\frac{v_{0} \alpha_{s 0}}{\alpha_{s}} t_{\mathrm{dis}}$.

The dimensionless form can be obtained as follows:

$\frac{h_{e}}{d_{s}}=\frac{v_{0} \alpha_{s 0}}{d_{s} \alpha_{s}} t_{\mathrm{dis}}$.

To sum up, the errors incurred in Eq. (36) may derive from the following aspects:

(i) If $t_{\text {dis }}$ and $t_{\text {exp }}$ have the same order of magnitude, Eq. (36) is no longer applicable. In addition, the closer the magnitudes of the two times are, the greater the error of Eq. (36).

(ii) It is assumed that when the mixture enters the pipe, the temperature of the particles rises to the maximum, ignoring the heat transfer process between the particles and water.

(iii) The influence of gas generated by dissociation on solid particles expansion is neglected.

(iv) Eq. (25) only applies for $v_{0}<v_{t}$; therefore, Eq. (36) is not applicable when $v_{0} \geq v_{t}$.

(v) The change of solid particle density is neglected in the process of hydrate dissociation.

\subsection{Applicability of the analytical expression}

To test the applicability of Eq. (36), the values of $h_{e} / d_{s}$ predicted by Eq. (36) against the numerical simulated results for various dimensionless numbers were plotted as shown in Figs. 14 and 16-20.

Fig. 14 shows the comparison between the numerical data for $h_{e} / d_{s}$ and Eq. (36) for various values of $R e_{s} / A r_{s}$. The values predicted by Eq. (36) were in good agreement with the numerical data for $1.56 \times 10^{-3} \leq$ $R e_{s} / A r_{s} \leq 2.45 \times 10^{-3}$, with error less than $25 \%$. However, the deviation between the predicted and the simulated values was large for $R e_{s} /$ $A r_{s}<1.56 \times 10^{-3}$ and $R e_{s} / A r_{s}>2.45 \times 10^{-3}$, indicating that Eq. (36) is not applicable to this condition. The decoupling conditions of hydrate dissociation and solid-liquid flow were all satisfied for the various $R e_{s}$ / $A r_{s}$ values listed in Table 3. For example, the ratios of the two characteristic times were $t_{\exp } / t_{\mathrm{dis}}=2.17 \times 10^{-3}$ for $R e_{s} / A r_{s}=7.80 \times 10^{-4}$ and $t_{\text {exp }} / t_{\text {dis }}=1.05 \times 10^{-4}$ for $R e_{s} / A r_{s}=2.90 \times 10^{-3}$. The reason why Eq. (36) was not applicable may be that the solid volume fraction $\alpha_{s}$ obtained from Eq. (25) was not accurate for $R e_{s} / A r_{s}<1.56 \times 10^{-3}$ and $R e_{s} / A r_{s}>2.45 \times 10^{-3}$.

In the derivation of $t_{\mathrm{dis}}$, the heat transfer process between the particles and water was ignored, assuming $T_{\mathrm{GH}}=T_{10}$. This hypothesis was verified as follows. For simplicity, the problem of heat transfer between a single solid particle and water with convective heat transfer as boundary conditions was considered. The initial temperature of the particle was $T_{s 0}$, and the temperature of the surrounding water was $T_{l 0}$. The velocity difference between the particle and the water was $\Delta v$. To estimate the time for heat conduction $t_{\text {cond }}$, it was assumed that heat transfer occurred only in the axial direction, as shown in Fig. 15. The heat conduction equation is expressed as follows (Bergman et al., 2011):

$\frac{\partial^{2} T}{\partial r^{2}}=\frac{1}{\kappa_{s}} \frac{\partial T}{\partial r}$

where $\kappa_{s}$ is the thermal diffusivity, $\kappa_{s}=\lambda_{s} / \rho_{s} c_{s}$. The initial condition is:

$T(r, 0)=T_{s 0}$,

and the boundary conditions are:

$\left.\frac{\partial T}{\partial r}\right|_{r=0}=0$,

and

$-\left.\lambda_{s} \frac{\partial T}{\partial r}\right|_{r=\frac{d_{s}}{2}}=h\left[\left(T\left(\frac{d_{s}}{2}, t\right)-T_{l 0}\right)\right]$,

where $h$ is the convective heat transfer coefficient, $h=\lambda_{l} N u_{s} / d_{s}$. Knudsen and Katz. (1958) expression for $N u_{s}$ is given by:

$N u_{s}=2+0.6 \operatorname{Pr}^{1 / 3} \operatorname{Re}_{s}^{1 / 2}$.

The dimensionless dependent variables are defined as $\theta_{*}=(T-$ 
$\left.T_{l 0}\right) /\left(T_{s 0}-T_{l 0}\right)$. A dimensional spatial coordinate is defined as $r_{*}=$ $2 r / d_{s}$, and a dimensionless time is defined as $t_{*}=4 \kappa_{s} t / d_{s}^{2}=F o$, where $F o$ is the Fourier number. Substituting $\theta_{*}, r_{*}$, and $t_{*}$ into (37)-(40), the heat equation becomes:

$\left.\begin{array}{l}\frac{\partial^{2} \theta_{*}}{\partial r_{*}^{2}}=\frac{\partial \theta_{*}}{\partial t_{*}} \\ \theta_{*}\left(r_{*}, \quad 0\right)=1 \\ \left.\frac{\partial \theta_{*}}{\partial r_{*}}\right|_{r_{*}=0}=0 \\ \left.\frac{\partial \theta_{*}}{\partial r_{*}}\right|_{r_{*}=1}=-\operatorname{Bi\theta } \theta_{*}\left(1, \quad t_{*}\right)\end{array}\right\}$,

where $B i$ is the Biot number, $B i=h d_{s} / 2 \lambda_{s}$. The exact solution of the above equation is an infinite series solution, which can be approximated by its first term for $F o>0.2$. The one-term approximation is (Bergman et al., 2011):

$\theta_{*}=\theta_{*} \frac{1}{\zeta r_{*}} \sin \left(\zeta r_{*}\right)$

where $\theta_{* 0}$ is the center temperature of the particle,

$\theta_{* 0}=\delta \exp \left(-\zeta^{2} t_{*}\right)$,

and $\zeta$ and $\delta$ are the coefficients determined by $B i$.

In the present work, it was assumed that the time $t_{w}$ needed for the particle center temperature to reach $99 \% T_{l 0}$ was equal to the time $t_{\text {cond }}$ needed to increase the particle temperature from $T_{s 0}$ to $T_{l 0}$. The time $t_{w}$ at which $T\left(0, t_{w}\right)=99 \% T_{10}$ can be obtained by Eq. (44). For the base case, $T_{s 0}=280.15 \mathrm{~K}, T_{l 0}=295.15 \mathrm{~K}$, and $\left(T_{l 0}-T_{s 0}\right) / T_{s 0}=0.054$; $B i=4.5$, and therefore $\zeta=2.513 \mathrm{rad}$ and $\delta=1.754$ (Bergman et al., 2011). Substituting $\zeta$ and $\delta$ into Eq. (44), $t_{*}=0.344$ can be obtained; thus, $t_{w}=t_{\text {cond }}=t_{*} d_{s}^{2} / 4 \kappa_{s}=1.42 \mathrm{~s}$. The use of the one-term approximation is appropriate with $t * 0.2$. Thus, the ratio of $t_{\text {cond }}$ and $t_{\text {dis }}$ is expressed as:

$\frac{t_{\text {cond }}}{t_{\text {dis }}}=\frac{1.42 \mathrm{~s}}{50.89 \mathrm{~s}}=2.79 \times 10^{-2}$.

Because temperature variation due to heat conduction occurs faster than hydrate dissociation, heat conduction in the particle and hydrate dissociation can be viewed as being decoupled. Thus, the assumption that the particle temperature rises quickly from $T_{s 0}$ to $T_{l 0}$ can be considered reasonable. It is noted that the value of $t_{\text {cond }} / t_{\text {dis }}$ increases with the increase of $\left(T_{l 0}-T_{s 0}\right) / T_{s 0}$; that is, the time of heat conduction is close to the time of hydrate dissociation. For example, when $T_{l 0}=$ $310.15 \mathrm{~K}$ and $\left(T_{l 0}-T_{s 0}\right) / T_{s 0}=0.107$, the values of $t_{\text {cond }}$ and $t_{\text {dis }}$ are $1.85 \mathrm{~s}$ and $7 \mathrm{~s}$ according to Eq. (44) and Eq. (33), respectively; therefore, $t_{\text {cond }} / t_{\text {dis }}=2.64 \times 10^{-1}$. Thus, the error of Eq. (36) becomes larger. In Fig. 16, the numerical data for $h_{e} / d_{s}$ are compared with the results of Eq. (36) for various values of $\left(T_{l 0}-T_{s 0}\right) / T_{s 0}$. The deviation between simulated and calculated results increases as $\left(T_{l 0}-T_{s 0}\right) / T_{s 0}$ increases, and the error increases from $1.22 \%$ to $23.66 \%$.

As shown in Fig. 17, the predicted values are generally larger than the simulated results for various values of $\varphi S_{H}$. The deviation between simulated and calculated results decreases as $\varphi S_{H}$ increases, and the error decreases from $15.35 \%$ to $2.49 \%$. The main reason is that the difference in hydrate saturation $S_{H}$ can change the thermodynamic properties of the solid particles. The thermal diffusivity of solid particles $\kappa_{s}=\lambda_{s} / \rho_{s} c_{s}$ decreases with the decrease of $S_{H}$; this means that the time of heat conduction $t_{\text {cond }}=t_{*} d_{s}^{2} / 4 \kappa_{s}$ increases. Moreover, the time of hydrate dissociation $t_{\mathrm{dis}}$ also decreases with decreasing $S_{H}$. Thus, $t_{\text {cond }}$ becomes closer to $t_{\text {dis }}$ as $S_{H}$ decreases, and the deviation between the simulated and calculated results increases.

Comparisons between the values predicted by Eq. (36) and the simulated results for $h_{e} / d_{s}$ at various values of $\alpha_{s 0}$ and $d_{s} / D$ are summarized in Figs. 18 and 19. The predicted values are all in good agreement with the simulated results for the various values of $\alpha_{s 0}$ and $d_{s} / D$, and the limiting errors are less than $5 \%$.

Eq. (36) also does not account for the effect of $d_{g} / d_{s}$ on $h_{e} / d_{s}$. Fig. 20 shows comparisons between the simulation data and predictions by Eq. (36) with different $d_{g} / d_{s}$ conditions. The variation range of $d_{g} / d_{s}$ is $0.03 \leq d_{g} / d_{s} \leq 1.67$. The results show that Eq. (36) is valid for $0.06 \leq d_{g} / d_{s} \leq 1.67$; the two results have error of less than $10 \%$.

\section{Conclusion}

In this study, the physical processes of multiphase flow containing GH dissociation were investigated based on the controlling dimensionless numbers derived by dimensional analysis. Without considering changes in material parameters during transportation, five main dimensionless numbers were obtained: $\operatorname{Re}_{s} / A r_{s},\left(T_{l 0}-T_{s 0}\right) / T_{s 0}, \alpha_{s 0}$, $\varphi S_{H}$, and $d_{s} / D$. The effects of these dimensionless parameters on multiphase flow in a pipe were then examined.

During transportation of GHBS particles upward in the pipe, after a period of time, the dissociation completion height $h$ was ultimately independent of time, denoted by the dissociation equilibrium height $h_{e}$. Based on the numerical simulation results, the power-law correlations for the dissociation equilibrium height $h_{e} / d_{s}$ and the friction coefficient of the pipe $c_{d}$ when the system was in a stable state were obtained using the curve-fitting method. It was found that $h_{e} / d_{s}$ increased with larger $R e_{s} / A r_{s}, \alpha_{s 0}$, and $\varphi S_{H}$, or with smaller $\left(T_{l 0}-T_{s 0}\right) / T_{s 0}$, whereas the variance of $d_{s} / D$ had almost no influence on the value of $h_{e} / d_{s}$. The value of $c_{d}$ was only dependent on $R e_{s} / A r_{s}$ and $\alpha_{s 0}$, increasing with decrease in $R e_{s} / A r_{s}$ and $\alpha_{s 0}$. The applicability of the power-law correlations in different $d_{g} / d_{s}$ cases was also studied, and the results showed that $h_{e} / d_{s}$ became smaller and $c_{d}$ tended to increase slowly with the increase in $d_{g} / d_{s}$. Better agreement between the numerical data and values predicted by the correlations was obtained when $0.06 \leq d_{g} / d_{s} \leq 1.67$ for $h_{e} / d_{s}$ and $0.03 \leq d_{g} / d_{s} \leq 0.67$ for $c_{d}$.

The characteristic time of $\mathrm{GH}$ dissociation was three to four orders of magnitude slower than that of the change of the top height of solid particles. The analytical expression for $h_{e} / d_{s}$ was obtained by decoupling hydrate dissociation and solid-liquid flow. First, the time required for the complete dissociation of hydrate was calculated. Then, the calculated height of the solid particles flowing upward with the range of $t_{\text {dis }}$ was represented by the dissociation equilibrium height $h_{e}$. The obtained dimensionless form of the expression is $h_{e} / d_{s}=v_{0} \alpha_{s 0} t_{\mathrm{dis}} / d_{s} \alpha_{s}$. The values predicted by the analytical expression were in good agreement with the numerical data for $1.56 \times 10^{-3} \leq R e_{s} / A r_{s} \leq 2.45 \times 10^{-3}$ and various values of $\left(T_{l 0}-T_{s 0}\right) / T_{s 0}, \alpha_{s 0}, \varphi S_{H}$, and $d_{s} / D$ within the scope of this study. The deviation between the predicted values and the simulated values gradually increased with increasing $\left(T_{l 0}-T_{s 0}\right) / T_{s 0}$ and decreasing $\varphi S_{H}$. The consistency of the power-law correlation and the analytic expression for dissociation equilibrium height within a specific parameter range can explain the correctness of the numerical simulation results.

In the mechanical-thermal exploitation method, the dissociation equilibrium height determines the installation position of the multiphase separation system, and the friction coefficient has an important influence on energy loss in the process of hydraulic transportation. In practical engineering design, the model for the dissociation equilibrium height and the friction coefficient obtained in this study can provide guidance for parameter design for optimal working conditions. The parameters to be optimized include $\operatorname{Re}_{s} / A r_{s}, d_{s} / D$, and $\alpha_{s 0}$. The conclusions of this paper elucidate the quantitative influence of the parameters to be optimized on the dissociation equilibrium height and friction coefficient. 


\section{Declaration of competing interest}

The authors declare that they have no known competing financial interests or personal relationships that could have appeared to influence the work reported in this paper.

\section{CRediT authorship contribution statement}

Peng Li: Conceptualization, Formal analysis, Investigation, Methodology, Validation, Visualization, Writing - original draft, Writing review \& editing. Xuhui Zhang: Conceptualization, Formal analysis, Project administration, Supervision, Writing - review \& editing. Xiaobing Lu: Conceptualization, Formal analysis, Supervision, Writing review \& editing.

\section{Acknowledgments}

This study is supported by the National Natural Science Foundation of China (No. 51639008, 11872365), the Youth Innovation Promotion Association of Chinese Academy of Sciences (No. 2017027), and the Key Special Project for Introduced Talents Team of Southern Marine Science and Engineering Guangdong Laboratory (Guangzhou) (No. GML2019ZD0307).

\section{Appendix A. Supplementary data}

Supplementary data to this article can be found online at https://doi. org/10.1016/j.jngse.2020.103470.

\section{Nomenclature}

$a_{1}, a_{2} \quad$ pre-factor

$A \quad$ pre-exponential factor

Ar Archimedes number

$B i \quad$ Biot number

Bo Bond number

c specific heat $\left(\mathrm{J} \mathrm{kg}^{-1} \mathrm{~K}^{-1}\right)$

$c_{d} \quad$ friction coefficient

C concentration $\left(\mathrm{kmol} \mathrm{m}^{-3}\right)$

d diameter $(\mathrm{m})$

$D \quad$ pipe diameter (m)

$E_{a} \quad$ activation energy $\left(\mathrm{J} \mathrm{mol}^{-1}\right)$

Fo Fourier number

$g \quad$ gravitational acceleration $\left(\mathrm{m} \mathrm{s}^{-2}\right)$

$h$ height $(\mathrm{m}) /$ convection heat transfer coefficient $\left(\mathrm{W} \mathrm{m}^{2} \mathrm{~K}^{-1}\right)$

$H \quad$ pipe height (m)

$\mathrm{Ja} \quad$ Jakob number

$k \quad$ rate constant

$M \quad$ molar mass $\left(\mathrm{kg} \mathrm{koml}^{-1}\right) /$ mass $(\mathrm{kg})$

$n \quad$ expansion index

$\mathrm{Nu} \quad$ Nusselt number

$r \quad$ reaction rate of hydrate dissociation $\left(\mathrm{kmol} \mathrm{m}^{-3} \mathrm{~s}^{-1}\right) /$ radius (m)

$R \quad$ universal gas constant $\left(\mathrm{J} \mathrm{mol}^{-1} \mathrm{~K}^{-1}\right)$

Re Reynolds number

$S \quad$ interphase mass source term $\left(\mathrm{kg} \mathrm{m}^{-3} \mathrm{~s}^{-1}\right)$

$S_{H} \quad$ hydrate saturation

$t \quad$ time (s)

$T \quad$ temperature (K)

$P \quad$ pressure $(\mathrm{Pa})$

$\mathrm{Pr} \quad$ Prandtl number

$v \quad$ velocity $\left(\mathrm{m} \mathrm{s}^{-1}\right)$

$Y \quad$ mass fraction

\section{Greek Letters}

$\alpha \quad$ volume fraction $\beta \quad$ temperature exponent

$\Delta H \quad$ latent heat of dissociation $\left(\mathrm{J} \mathrm{mol}^{-1}\right)$

$\Delta p \quad$ pressure drop $(\mathrm{Pa})$

$\eta \quad$ rate exponent

$\kappa \quad$ thermal diffusivity $\left(\mathrm{m}^{2} \mathrm{~s}^{-1}\right)$

$\lambda$ thermal conductivity $\left(\mathrm{W} \mathrm{m}^{-1} \mathrm{~K}^{-1}\right)$

$\mu \quad$ shear viscosity (Pa s)

$\rho$ density $\left(\mathrm{kg} \mathrm{m}^{-3}\right)$

$\sigma \quad$ surface tension coefficient $\left(\mathrm{N} \mathrm{m}^{-1}\right)$

$\tau \quad$ friction drag per pipe wall unit area (Pa)

$\varphi \quad$ porosity

\section{Subscripts}

$\begin{array}{ll}\text { c } & \text { characteristic } \\ \text { cond } & \text { conduction } \\ \text { dis } & \text { dissociation } \\ \mathrm{e} & \text { equilibrium } \\ \text { exp } & \text { expansion } \\ i & \text { component } \\ g & \text { gas phase } \\ \mathrm{GH} & \text { gas hydrate } \\ l & \text { liquid phase } \\ m & \text { mixture } \\ s & \text { solid phase } \\ t & \text { terminal }\end{array}$

\section{Superscripts}

$b, c, d, e, f$ fitting exponent

\section{References}

Bai, Y.H., Li, Q.P., Yu, X.C., et al., 2007. Numerical study on dissociation of gas hydrate of gas hydrate and its sensitivity to physical parameters. China Ocean Eng. 21 (4), 625-636.

Bergman, T.L., Lavine, A.S., Incorpera, F.P., et al., 2011. Fundamentals of Heat and Mass Transfer. John Wiley \& Sons.

Collett, T.S., Lewis, R.E., Winters, W.J., et al., 2011. Downhole well log and core montages from the mount elbert gas hydrate stratigraphic test well, Alaska North Slope. Mar. Petrol. Geol. 28 (2), 561-577.

Dickens, G.R., Quinby, H.M.S., 1994. Methane hydrate stability in seawater. Geophys. Res. Lett. 21 (19), 2115-2118.

Friend, D.G., Ely, J.F., Ingham, H., 1989. Thermo-physical properties of methane. J. Phys. Chem. Ref. Data 18 (2), 583-638.

Fujii, T., Noguchi, S., Takayama, T., et al., 2013. Site Selection and Formation Evaluation at the $1^{\text {st }}$ Offshore Methane Hydrate Production Test Site in the Eastern Nankai through. $75^{\text {th }}$ EAGE Conference \& Exhibition incorporating SPE EUROPEC 2013, London.

Haider, A., Levenspiel, O., 1989. Drag coefficient and terminal velocity of spherical and nonspherical particles. Power Technol. 58, 63-70.

Khan, A.R., Richardson, J.F., 1989. Fluid-particle interactions and flow characteristics of fluidized beds and settling suspensions of spherical particles. Chem. Eng. Commun. $78,111-130$.

Knudsen, J.G., Katz, D.L., 1958. Fluid Mechanics and Heat Transfer. McGraw-Hill, New York.

Li, P., Zhang, X.H., Lu, X.B., 2018. Numerical simulation on solid-liquid two-phase flow in cross fractures. Chem. Eng. Sci. 181, 1-18.

Li, P., Zhang, X.H., Lu, X.B., 2019. Three-dimensional Eulerian modeling of gas-liquidsolid flow with gas hydrate dissociation in a vertical pipe. Chem. Eng. Sci. 196, 145-165.

Makogon, Y.F., Holditch, S.A., Makogon, T.Y., 2005. Russian field illustrates gas-hydrate production. Oil Gas J. 103, 43-47.

Makogon, Y.F., Omelchenko, R.Y., 2013. Commercial gas production from Messoyakha deposits in hydrate conditions. J. Nat. Gas Sci. Eng. 11, 1-6.

Moridis, G.J., Collett, T.S., Boswell, R., et al., 2009. Toward production from gas hydrates: current status, assessment of resources, and simulation-based evaluation of technology and potential. SPE Reservoir Eval. Eng. 12 (5), 745-751.

Richardson, J.F., Zaki, W.N., 1954. Sedimentation and fluidization: Part I. Trans. Inst. Chem. Eng. 32, 35-53.

Sean, W.Y., Sato, T., Yamasaki, A., et al., 2007. CFD and experimental study on methane hydrate dissociation Part I. Dissociation under water flow. AIChE J. 53, 262-274.

Silva, J.M.D., Dawe, R., 2011. Towards commercial gas production from hydrate deposits. Energies 4, 215-238.

Tan, Q.M., 2011. Dimensional Analysis: with Case Studies in Mechanics. Springer Verlag, Berlin, Heidelberg.

Wagner, W., Pruss, A., 2002. The IAPWS formulation 1995 for the thermodynamic properties of ordinary water substance for general and scientific use. J. Phys. Chem. Ref. Data 31 (2), 387-535. 
Wang, G.R., Zhong, L., Zhou, S.W., et al., 2017. Jet breaking tools for natural gas hydrate exploitation and their support technologies. Nat. Gas. Ind. 12, 68-74.

White, M.D., Wurstner, S.K., McGrail, B.P., 2011. Numerical studies of methane production from Class 1 gas hydrate accumulation enhanced with carbon dioxide injection. Mar. Petrol. Geol. 28 (2), 546-560.

Wu, K.S., Jia, T.W., Lian, D., et al., 2017. Research on design of mining tools of marine gas hydrates reservoirs. Mech. Sci. Technol. Aerospace Eng. (in Chinese) 36 (2), 225-231.
Zhang, X.H., Lu, X.B., 2016. A new exploitation method for gas hydrate in shallow stratum: mechanical-thermal method (in Chinese). Chin. J. Theor. Appl. Mech. 48 (5), 1238-1246.

Zhang, X.H., Lu, X.B., Liu, L.L., 2014. Advances in natural gas hydrate recovery methods. Prog. Geophys, 29 (2), 858-869.

Zhong, W., Yu, A., Zhou, G., et al., 2016. CFD simulation of dense particulate reaction system: approaches, recent advances and applications. Chem. Eng. Sci. 140, 16-43. 